\title{
NUMERICAL STUDY OF GAMMA-RAY BURST JET FORMATION IN COLLAPSARS
}

\author{
Shigehiro Nagataki, ${ }^{1,2}$ Rohta Takahashi, ${ }^{3}$ Akira Mizuta, ${ }^{4}$ and Tomoya Takiwaki ${ }^{5}$
}

\begin{abstract}
Two-dimensional MHD simulations are performed using the ZEUS-2D code to investigate the dynamics of a collapsar that generates a GRB jet, taking account of realistic equation of state, neutrino cooling and heating processes, magnetic fields, and gravitational force from the central black hole and self-gravity. It is found that neutrino heating processes are not efficient enough to launch a jet in this study. It is also found that a jet is launched mainly by $B_{\phi}$ fields that are amplified by the winding-up effect. However, since the ratio of total energy relative to the rest-mass energy in the jet is not as high as several hundred, we conclude that the jets seen in this study are not GRB jets. This result suggests that general relativistic effects will be important to generating a GRB jet. Also, the accretion disk with magnetic fields may still play an important role in launching a GRB jet, although a simulation for much longer physical time $(\sim 10-100 \mathrm{~s})$ is required to confirm this effect. It is shown that a considerable amount of ${ }^{56} \mathrm{Ni}$ is synthesized in the accretion disk. Thus, there will be a possibility for the accretion disk to supply the sufficient amount of ${ }^{56} \mathrm{Ni}$ required to explain the luminosity of a hypernova. Also, it is shown that neutron-rich matter due to electron captures with high entropy per baryon is ejected along the polar axis. Thus, there will be a possibility that $r$-process nucleosynthesis occurs at such a region. Finally, many neutrons will be ejected from the jet, which suggests that signals from the neutron decays may be observed as the delayed bump of the light curve of the afterglow or gamma rays.
\end{abstract}

Subject headings: accretion, accretion disks — black hole physics — gamma rays: bursts — MHD nuclear reactions, nucleosynthesis, abundances - supernovae: general

\section{INTRODUCTION}

There has been growing evidence linking long gamma-ray bursts (GRBs; in this study we consider only long GRBs, so we refer to long GRBs as GRBs hereafter for simplicity) to the death of massive stars. The host galaxies of GRBs are star-forming galaxies, and the positions of GRBs appear to trace the blue light of young stars (Vreeswijk et al. 2001; Bloom et al. 2002; Gorosabel et al. 2003). Also, "bumps" observed in some afterglows can be naturally explained as the contribution of bright supernovae (Bloom et al. 1999; Reichart 1999; Galama et al. 2000; Garnavich et al. 2003). Moreover, direct evidence of some GRBs accompanied by supernovae has been reported, such as the association of GRB 980425 with SN 1998bw (Galama et al. 1998; Iwamoto et al. 1998), that of GRB 030329 with SN 2003dh (Hjorth et al. 2003; Price et al. 2003; Stanek et al. 2003), and that of GRB 060218 with SN 2006aj (Mirabal et al. 2006; Mazzali et al. 2006).

It should be noted that these supernovae (except for SN 2006aj) are categorized as a new type of supernovae with large kinetic energy $\left(\sim 10^{52} \mathrm{ergs}\right)$, nickel mass $\left(\sim 0.5 M_{\odot}\right)$, and luminosity (Iwamoto et al. 1998; Woosley et al. 1999), so these supernovae are sometimes called hypernovae. The total explosion energy of the order of $10^{52}$ ergs is too important not to be emphasized because it is generally believed that a normal core-collapse supernova cannot cause such an energetic explosion. Thus, another scenario has to be considered to explain the system of a GRB associated with a hypernova. One of the most promising scenarios

\footnotetext{
1 Yukawa Institute for Theoretical Physics, Kyoto University, Sakyo-ku, Kyoto 606-8502, Japan; nagataki@yukawa.kyoto-u .ac.jp.

2 KIPAC, Stanford University, Stanford, CA 94309.

3 Graduate School of Arts and Sciences, University of Tokyo, Tokyo 153 8902, Japan.

4 Max-Planck-Institute für Astrophysik, 85741 Garching, Germany.

5 Department of Physics, University of Tokyo, Bunkyo-ku, Tokyo 113-0033,
}

is the collapsar scenario (Woosley 1993). In the collapsar scenario, a black hole is formed as a result of gravitational collapse. Also, rotation of the progenitor plays an essential role. Due to the rotation, an accretion disk is formed around the equatorial plane. On the other hand, the matter around the rotation axis freely falls into the black hole. MacFadyen \& Woosley (1999) pointed out that the jet-induced explosion along the rotation axis may occur due to the heating through neutrino-antineutrino pair annihilation that is emitted from the accretion disk (see also Fryer \& Mészáros 2003).

It is true that the collapsar scenario is the breakthrough on the problem of the central engine of GRBs. However, there are many effects that have to be involved in order to establish the scenario firmly. First of all, neutrino heating effects have to be investigated carefully by including microphysics of neutrino processes in the numerical simulations. It is true that MacFadyen \& Woosley (1999) have performed the numerical simulations of the collapsar, in which a jet is launched along the rotation axis (see also Aloy et al. 2000 ), but detailed microphysics of neutrino heating is not included in their simulations. Secondly, it was pointed out that effects of magnetic fields and rotation may play an important role in launching the GRB jets (Proga et al. 2003; Mizuno et al. 2004a, 2004b; Proga 2005; Shibata \& Sekiguchi 2005; Sekiguchi \& Shibata 2005; Fujimoto et al. 2006), although neutrino heating effects are not included in their works. Recently, Rockefeller et al. (2006) presented three-dimensional simulations of collapsars with the smoothed particle hydrodynamics code. In their study, the three-flavor flux-limited diffusion package is used to take into account neutrino cooling and absorption of electron-type neutrinos, although neutrino-antineutrino pair annihilation is not included. They have shown that $\alpha$-viscosity drives energetic explosion through three-dimensional instabilities and angular momentum transfer, although the jet is not launched and magnetic fields (source of the viscosity) are not included in their study. Thus, it is not clear which effects are most important for launching a 
GRB jet, that is, what process is essential as the central engine of GRBs.

Due to the motivation mentioned above, we have performed two-dimensional magnetohydrodynamic (MHD) simulations of collapsars with magnetic fields, rotation, and neutrino cooling/ heating processes. In our simulations, the realistic equation of state (EOS) of Blinnikov et al. (1996) and effects of photodisintegration of nuclei are also included. We investigated the influence of magnetic fields on the dynamics of collapsars by changing the initial amplitude of the magnetic fields. In $\S 2$ models and numerical methods are explained. Results are shown in $\S 3$. Discussions are described in $\S 4$. Summary and conclusions are presented in $\S 5$.

\section{MODELS AND NUMERICAL METHODS}

Our models and numerical methods of simulations in this study are shown in this section. First, we present equations of ideal MHD, then initial and boundary conditions are explained. Microphysics included in this study (EOS, nuclear reactions, and neutrino processes) is also explained.

\subsection{Magnetohydrodynamics}

We have performed two-dimensional MHD simulations taking account of self-gravity and gravitational potential of the central point mass. The calculated region corresponds to a quarter of the meridian plane under the assumption of axisymmetry and equatorial symmetry. The spherical mesh with $150(r) \times 30(\theta)$ grid points is used for all the computations. The radial grid is nonuniform, extending from $r=1.0 \times 10^{6}$ to $1.0 \times 10^{10} \mathrm{~cm}$ with finer grids near the center, while the polar grid is uniform. The minimum radial grid is set to be $3.0 \times 10^{5} \mathrm{~cm}$. We have confirmed that a free flow is smoothly solved with this grid resolution.

The basic equations in the following form are finite-differenced on the spherical coordinates:

$$
\begin{gathered}
\frac{D \rho}{D t}=-\rho \nabla \cdot \boldsymbol{v} \\
\rho \frac{D \boldsymbol{v}}{D t}=-\nabla p-\rho \nabla\left(\Phi_{p}+\Phi_{s}\right)+\frac{1}{4 \pi}(\nabla \times \boldsymbol{B}) \times \boldsymbol{B}, \\
\rho \frac{D}{D t}\left(\frac{e}{\rho}\right)=-p \nabla \cdot \boldsymbol{v}-L_{\nu}^{-}+L_{\nu}^{+}+L_{\mathrm{nucl}}, \\
\frac{\partial \boldsymbol{B}}{\partial t}=\nabla \times(\boldsymbol{v} \times \boldsymbol{B}), \\
\Delta \Phi_{s}=4 \pi G \rho, \\
\frac{D Y_{e}}{D t}=-Y_{p} \Gamma_{p \rightarrow n}+Y_{n} \Gamma_{n \rightarrow p},
\end{gathered}
$$

where $\rho, \boldsymbol{v}, p, \Phi_{p}, \Phi_{s}, e, L_{\nu}^{ \pm}, L_{\text {nucl }}, Y_{e}, Y_{p}, Y_{n}, \Gamma_{p \rightarrow n}$, and $\Gamma_{n \rightarrow p}$ are density, velocity, pressure, gravitational potential due to the central point mass (black hole), gravitational potential due to selfgravity, internal energy density, heating/cooling rates due to neutrino processes, energy gain (loss) rate due to nuclear reaction, and fraction of electron, proton, neutron, and reaction rate from proton to neutron (electron capture rate plus $\nu_{e}$ capture on neutron) and from neutron to proton (positron capture plus $\bar{\nu}_{e}$ capture on proton), respectively. The Lagrangian derivative is denoted as $D / D t$. The gravitational potential of the black hole is modified to take into account some of the general relativistic effects (Paczyński $\&$ Witta 1980), $\Phi_{p}=-G M /\left(r-r_{\mathrm{S}}\right)$, where $r_{\mathrm{S}}=2 G M / c^{2}$ is the Schwarzschild radius. Self-gravity is obtained in the ZEUS-2D code developed by Stone \& Norman (1992a, 1992b), by solving the matrix that results from finite-differencing the Poisson equa- tion in two dimensions. The ZEUS-2D code is also used to solve the MHD equations with second-order accurate interpolation in space.

Energy gain (loss) rate due to nuclear reaction and heating/ cooling rates due to neutrino processes are described in $\S \S 2.3 .2$ and 2.3.3. Effects of $\alpha$-viscosity and (anomalous) resistivity are not included in this study to avoid the uncertainty of the treatment of these effects.

\subsection{Initial and Boundary Conditions}

We adopt the model E25 in Heger et al. (2000). This model corresponds to a star that has $25 M_{\odot}$ initially with solar metallicity but loses its mass and becomes $5.45 M_{\odot}$ of a Wolf-Rayet star at the final stage. This model seems to be a good candidate as a progenitor of a GRB since losing their envelope will be suitable to be a Type Ic-like supernova and to make a baryon-poor fireball. The mass of the iron core is $1.69 M_{\odot}$ in this model. Thus, we assume that the iron core has collapsed and formed a black hole at the center. This treatment is the same as in Proga et al. (2003). The Schwarzschild radius of the black hole is $5.0 \times 10^{5} \mathrm{~cm}$ initially.

We explain how the angular momentum is distributed initially. At first, we performed a one-dimensional simulation for the spherical collapse of the progenitor for $0.1 \mathrm{~s}$ when the innermost Si layer falls to the innermost boundary $\left(=10^{6} \mathrm{~cm}\right)$. After the spherical collapse, angular momentum was distributed so as to provide a constant ratio of 0.05 of centrifugal force to the component of gravitational force perpendicular to the rotation axis at all angles and radii, except where that prescription resulted in $j_{16}$ greater than a prescribed maximum value, 10 . This treatment is similar to the one in MacFadyen \& Woosley (1999). The total initial rotation energy is $2.44 \times 10^{49} \mathrm{ergs}$, which corresponds to $1.3 \times 10^{-2}$ for the initial ratio of the rotation energy to the gravitational energy $(T / W)$.

Configuration and amplitude of the magnetic fields in a progenitor prior to collapse are still uncertain. Thus, in this study we choose a simple form for the initial configuration prior to collapse and the amplitude is changed parametrically. Initial configuration of the magnetic fields is chosen as follows:

$$
\boldsymbol{B}(\boldsymbol{r})= \begin{cases}\frac{1}{3} B_{0}\left(\frac{r_{0}}{r}\right)^{3}\left(2 \cos \theta \boldsymbol{e}_{r}+\sin \theta \boldsymbol{e}_{\theta}\right), & r \geq r_{0}, \\ \frac{2}{3} B_{0}\left(\cos \theta \boldsymbol{e}_{r}-\sin \theta \boldsymbol{e}_{\theta}\right), & r<r_{0} .\end{cases}
$$

This configuration represents that the magnetic fields are uniform in a sphere $\left(r<r_{0}\right)$ and dipole outside of the sphere. We set $r_{0}$ to be the boundary between $\mathrm{CO}$ core and He layer $\left(=3.6 \times 10^{9} \mathrm{~cm}\right)$. $B_{0}$ corresponds to the strength of the magnetic field in the sphere. We have chosen $B_{0}$ to be $0,10^{8}, 10^{9}, 10^{10}, 10^{11}$, and $10^{12} \mathrm{G}$.

As for the boundary condition in the radial direction, we adopt the outflow boundary condition for the inner and outer boundaries. That is, the flow from the central black hole is prohibited at the inner boundary and the inflow from the surface of the progenitor is prohibited at the outer boundary. Of course, the mass of the central black hole becomes larger due to the mass accretion from the inner boundary. As for the boundary condition in the zenith angle direction, the axis of the symmetry boundary condition is adopted for the rotation axis, while the reflecting boundary condition is adopted for the equatorial plane. As for the magnetic fields, the equatorial symmetry boundary condition, in which the normal component is continuous and the tangential component is reflected, is adopted. 


\subsection{Microphysics}

\subsubsection{Equation of State}

The EOS used in this study is the one developed by Blinnikov et al. (1996). This EOS contains an electron-positron gas with arbitrary degeneracy, which is in thermal equilibrium with blackbody radiation and ideal gas of nuclei. We used the mean atomic weight of nuclei to estimate the ideal gas contribution to the total pressure, although its contribution is negligible relative to those of electron-positron gas and thermal radiation in our simulations.

\subsubsection{Nuclear Reactions}

Although the ideal gas contribution of nuclei to the total pressure is negligible, effects of energy gain/loss due to nuclear reactions are important. In this study, nuclear statistical equilibrium (NSE) was assumed for the region where $T \geq 5 \times 10^{9} \mathrm{~K}$ is satisfied. This treatment is based on the assumption that the timescale to reach and maintain NSE is much shorter than the hydrodynamical time. Note that complete Si burning occurs in explosive nucleosynthesis of core-collapse supernovae for the region $T \geq$ $5 \times 10^{9} \mathrm{~K}$ ( Thielemann et al. 1996). The hydrodynamical time in this study, $\sim 1 \mathrm{~s}$ (as shown in Figs. 3, 4, and 10 below, the nuclear reaction occurs at $\sim 10^{7}-10^{8} \mathrm{~cm}$ where the radial velocity is of the order of $10^{7}-10^{8} \mathrm{~cm} \mathrm{~s}^{-1}$ ), is comparable to the explosive nucleosynthesis in core-collapse supernovae, so the assumption of NSE adopted in this study seems to be good. Five nuclei, $n, p$, ${ }^{4} \mathrm{He},{ }^{16} \mathrm{O}$, and ${ }^{56} \mathrm{Ni}$, were used to estimate the binding energy of ideal gas of nuclei in NSE for given $\left(\rho, T, Y_{e}\right) . Y_{e}$ is the electron fraction that is obtained from the calculations of neutrino processes in $\S 2.3 .3$. On the other hand, we assumed that no nuclear reaction occurs for the region where $T<5 \times 10^{9} \mathrm{~K}$.

\subsubsection{Neutrino Processes}

Neutrino cooling processes due to pair capture on free nucleons, pair annihilation, and plasmon decay are included in this study. Since photoneutrino and bremsstrahlung processes are less important ones at $10^{9} \mathrm{~K}<T<10^{11} \mathrm{~K}$ and $\rho<10^{12} \mathrm{~g} \mathrm{~cm}^{-3}$ (Itoh et al. 1989), where neutrino cooling effects are important in our calculations, we do not include these processes.

As for the electron capture on free protons, we extend the formulation of Epstein \& Pethick (1981) to arbitrary degeneracy. The energy loss rate per proton $\dot{Q}_{\mathrm{EC}}\left(\mathrm{GeV} \mathrm{s}^{-1}\right.$ proton $\left.^{-1}\right)$ is

$$
\begin{aligned}
\dot{Q}_{\mathrm{EC}}= & \frac{G_{\beta}^{2}\left(C_{V}^{2}+3 G_{A}^{2}\right)}{2 \pi^{3} \hbar}\left[\left(k_{\mathrm{B}} T\right)^{6} F_{5}(x)+2 Q_{v}\left(k_{\mathrm{B}} T\right)^{5} F_{4}(x)\right. \\
& \left.+Q_{v}^{2}\left(k_{\mathrm{B}} T\right)^{4} F_{3}(x)\right] \frac{\exp \left(F_{5}(\eta) / F_{4}(\eta)\right)}{1+\exp \left(F_{5}(\eta) / F_{4}(\eta)\right)},
\end{aligned}
$$

where $G_{\beta}=G_{\mathrm{F}} \cos \theta_{\mathrm{C}}$ (in units of $\mathrm{GeV}^{-2}$ ) is the Fermi coupling constant ( $\theta_{\mathrm{C}}$ is the Cabbibo angle), $C_{V}=\frac{1}{2}+2 \sin ^{2} \theta_{\mathrm{W}}$ is the vector coupling constant ( $\theta_{\mathrm{W}}$ is the Weinberg angle), $C_{A}=\frac{1}{2}$ is the axial vector coupling constant, $k_{\mathrm{B}}$ is the Boltzmann constant, and $Q_{v}=\left(m_{n}-m_{p}\right) c^{2}$ is the mass difference between neutron and proton. Parameters $x$ and $\eta$ are defined as $x=\left(\mu_{e}-Q_{v}\right) /\left(k_{\mathrm{B}} T\right)$ and $\eta=\mu_{e} /\left(k_{\mathrm{B}} T\right)$, where $\mu_{e}$ is the chemical potential of electrons. $F_{i}(x)$ is the Fermi integral that is defined as

$$
F_{i}(x)=\int_{0}^{\infty} \frac{y^{k}}{1+e^{y-x}} d y .
$$

We used the formulation derived by Takahashi et al. (1978) to solve the Fermi integral. Note that the leading term becomes the first term in equation (9) and obeys $\propto T^{6}$ when electrons are not degenerated, although other terms dominate when electrons become degenerated. We also include the Fermi blocking factor (Herant et al. 1994) due to $\nu_{e}$ assuming that the chemical potential of $\nu_{e}$ is zero and the mean energy of the emitted neutrinos by this process is $k_{\mathrm{B}} T F_{5}(\eta) / F_{4}(\eta)$ (Rosswog \& Liebendörfer 2003). All we have to do is change $\mu_{e}$ to $-\mu_{e}$ and $Q_{v}$ to $-Q_{v}$ to estimate the positron capture on free neutrons. As for the plasmon decays, we used the formulation of Itoh et al. (1989) with the blocking factor (Herant et al. 1994) assuming that the chemical potential of $\nu_{e}$ is zero and the mean energy of the emitted neutrinos is $k_{\mathrm{B}} T\left[1+\gamma_{\text {loss }}^{2} / 2\left(1+\gamma_{\text {loss }}\right)\right]$, where $\gamma_{\text {loss }}=5.565 \times 10^{-2}\left(\pi^{2} / 3+\eta^{2}\right)^{1 / 2}$ (Rosswog \& Liebendörfer 2003). We have also used the formulation of Itoh et al. (1989) for pair annihilation with the blocking factor (Herant et al. 1994). The mean energies of $\nu_{e}$ and $\bar{\nu}_{e}$ emitted by this process are $k_{\mathrm{B}} T F_{4}(\eta) / F_{3}(\eta)$ and $k_{\mathrm{B}} T F_{4}(-\eta) / F_{3}(-\eta)$, respectively (Cooperstein et al. 1986).

In our simulations, density at the innermost region reaches as high as $10^{12} \mathrm{~g} \mathrm{~cm}^{-3}$, where nuclei are almost photodisintegrated into nucleons. In this region, as discussed in $\S 4.1$, the optically thin limit breaks down. In order to take into account such a highdensity region, a neutrino leakage scheme is introduced (Kotake et al. 2003). In this scheme, we calculate the typical diffusion timescale for neutrinos to escape from the system. Since chemical composition is dominated by nucleons at such a high-density region, the opacity is mainly determined by scattering and absorption by nucleons for $\nu_{e}$ and $\bar{\nu}_{e}$ and scattering by nucleons for $\nu_{\mu}, \bar{\nu}_{\mu}, \nu_{\tau}$, and $\bar{\nu}_{\tau}$. The mean free path can be written as

$$
\begin{gathered}
\lambda_{\nu_{e}}^{-1}=\frac{\rho}{m_{u}} \sigma_{\mathrm{sc}}\left(\epsilon_{\nu_{e}}\right)+\frac{\rho Y_{n}}{m_{u}} \sigma_{\mathrm{ab}}\left(\epsilon_{\nu_{e}}\right) \text { for } \nu_{e}, \\
\lambda_{\bar{\nu}_{e}}^{-1}=\frac{\rho}{m_{u}} \sigma_{\mathrm{sc}}\left(\epsilon_{\bar{\nu}_{e}}\right)+\frac{\rho Y_{p}}{m_{u}} \sigma_{\mathrm{ab}}\left(\epsilon_{\bar{\nu}_{e}}\right) \text { for } \bar{\nu}_{e}, \\
\lambda_{\nu_{x}}^{-1}=\frac{\rho}{m_{u}} \sigma_{\mathrm{sc}}\left(\epsilon_{\nu_{x}}\right) \quad \text { for other flavors }
\end{gathered}
$$

where $m_{u}, \sigma_{\mathrm{sc}}, \sigma_{\mathrm{ab}}, Y_{p}, Y_{n}, \epsilon_{\nu_{e}}, \epsilon_{\bar{\nu}_{e}}$, and $\epsilon_{\nu_{x}}$ are the atomic mass unit, scattering cross section by nucleon, absorption cross section by nucleon, mass fraction of proton and neutron, and mean energies of $\nu_{e}, \bar{\nu}_{e}$, and $\nu_{x}$, respectively. We take the cross sections for these interactions from Bethe (1990). The diffusion timescale is given by

$$
\tau_{\text {diff }}=\frac{3 \Delta R^{2}}{\pi^{2} c \lambda},
$$

where we take $\Delta R=(r \Delta r \Delta \theta)^{1 / 2}$ as the typical diffusion length. Parameters $\Delta r$ and $r \Delta \theta$ are grid sizes in the radial and polar directions at that point. Neutrino emissivity $\left(\mathrm{GeV} \mathrm{cm}^{-3} \mathrm{~s}^{-1}\right)$ at the point is calculated as

$$
f_{\nu}^{\text {leak }}=f_{\nu} \min \left(1.0, \frac{d t}{\tau_{\text {diff }}}\right),
$$

where $f_{\nu}$ and $d t$ are neutrino emissivity obtained by the optically thin limit and time step of the simulations, respectively. On the other hand, when we estimate the neutrino heating rate, we take the optically thin limit for all the regions for simplicity. This corresponds to the upper limit that we estimate for the neutrino heating effects. Even at this limit, as shown later, jetlike explosion does not occur by the neutrino heating (as shown in Fig. 1 below). Also, this optically thin limit will be justified since the neutrino cooling rate dominates the heating rate, as shown in Figure 8 below. Detailed discussion on this point is presented in $\S 4.1$. 
The neutrino heating process due to neutrino pair annihilation and $\nu_{e}$ and $\bar{\nu}_{e}$ captures on free nucleons with blocking factors of electrons and positrons are included in this study. As for the neutrino pair annihilation process, the formulation of Goodman et al. (1987) (with blocking factors) is adopted. The $\nu_{e}$ and $\bar{\nu}_{e}$ captures on free nucleons are inverse processes of electron/positron captures. The calculation of neutrino heating is the most expensive in the simulation of this study. Thus, to save the CPU time, the neutrino heating processes are calculated only within the limited regions $\left(r<10^{9} \mathrm{~cm}\right)$. Moreover, we adopt some criteria as follows in order to save CPU time. We have to determine the energy deposition regions and emission regions to estimate the neutrino heating rate. As for the neutrino pair annihilation process, we adopt no criterion for the energy deposition regions other than $r<10^{9} \mathrm{~cm}$, while the emission regions satisfy the criterion of $T \geq 3 \times 10^{9} \mathrm{~K}$. As for the neutrino capture process, we adopt the criteria as follows: The absorption regions should satisfy the criterion of $\rho \geq 10^{4} \mathrm{~g} \mathrm{~cm}^{-3}$, while the emission regions satisfy the criteria of (1) $\rho \geq 10^{3} \mathrm{~g} \mathrm{~cm}^{-3}$ and (2) $T \geq 10^{9} \mathrm{~K}$. Also, these heating rates had to be updated every 100 time steps to save CPU time. Of course, this treatment has to be improved in the future. However, this treatment seems to be justified as follows: The total time step is of the order of $10^{6}$ steps. The final physical time is of the order of seconds, so the typical time step is $\sim 10^{-6} \mathrm{~s}$. This means that the heating rate is updated every $\sim 10^{-4} \mathrm{~s}$, which will be shorter than the typical dynamical timescale. This point is discussed in detail in $\S 4.1$.

The heating process of neutrino pair annihilation is calculated as follows. Let points $\mathrm{A}$ and $\mathrm{B}$ be in the emission regions and point $\mathrm{C}$ be in the energy deposition regions. Let $d_{\mathrm{A}}, d_{\mathrm{B}}$, and $d_{\mathrm{C}}$ be distances between $\mathrm{A}$ and $\mathrm{B}, \mathrm{B}$ and $\mathrm{C}$, and $\mathrm{C}$ and $\mathrm{A}$, respectively. The angle $\psi$ is defined as the angle ACB. The energy deposition rate at $\mathrm{C}, \dot{Q}(C)\left(\mathrm{GeV} \mathrm{cm}^{-3} \mathrm{~s}^{-1}\right)$, is given formally by integrating the emission regions as

$$
\begin{aligned}
\dot{Q}(C)= & \frac{K G_{\mathrm{F}}^{2}}{2 \pi c} \iint d x_{\mathrm{A}}^{3} d x_{\mathrm{B}}^{3} \frac{(1-\cos \psi)^{2}}{d_{\mathrm{A}}^{2} d_{\mathrm{B}}^{2}} \\
& \times \int d \epsilon_{\nu} d \epsilon_{\bar{\nu}} \epsilon_{\nu} \epsilon_{\bar{\nu}}\left(\epsilon_{\nu}+\epsilon_{\bar{\nu}}\right) F_{\nu} F_{\bar{\nu}}\left(1-f_{e}^{-}\right)\left(1-f_{e}^{+}\right),
\end{aligned}
$$

where $K$ is $\left(1-4 \sin ^{2} \theta_{W}+8 \sin ^{4} \theta_{W}\right) /(6 \pi)$ for $\nu_{\mu} \nu_{\bar{\mu}}$ and $\nu_{\tau} \nu_{\bar{\tau}}$ annihilation and $\left(1+4 \sin ^{2} \theta_{W}+8 \sin ^{4} \theta_{W}\right) /(6 \pi)$ for $\nu_{e} \nu_{\bar{e}}$ annihilation (Goodman et al. 1987), $\boldsymbol{x}_{\mathrm{A}}$ and $\boldsymbol{x}_{\mathrm{B}}$ are locations of the points $\mathrm{A}$ and $\mathrm{B}, \epsilon_{\nu, \bar{\nu}}$ are energies of (anti)neutrinos, $F_{\nu, \bar{\nu}}$ are energy spectra $\left(\mathrm{cm}^{-3} \mathrm{~s}^{-1} \mathrm{GeV}^{-1}\right)$ of (anti)neutrinos, and $f_{e}^{ \pm}$are Fermi blocking factors of electrons/positrons at point $\mathrm{C}$. In this formulation, $G_{\mathrm{F}}$ is written in units of $\mathrm{cm} \mathrm{GeV}^{-1}$. It is easily seen that equation (15) is an eight-dimensional integral and it takes too much time to accomplish this integral. Thus, we have approximated that each neutrino energy spectrum due to each emission process is a monotonic one; that is, we assumed that only neutrinos with average energy are emitted when equation (15) is carried out. Since the energy loss rate $\left(\mathrm{GeV} \mathrm{cm} \mathrm{cm}^{-3} \mathrm{~s}^{-1}\right)$ is obtained from the formulations of the neutrino cooling process mentioned above, the number of emitted neutrinos for each process is calculated by dividing the average energy of emitted neutrinos for the process. Although the calculated region is a quarter of the meridian plane, the neutrino flux from the southern hemisphere is included assuming the symmetry relative to the equatorial plane. The same treatment with the neutrino pair annihilation process is adopted to estimate the heating rate due to $\nu_{e}$ and $\bar{\nu}_{e}$ capture processes;
TABLE 1

\begin{tabular}{|c|c|c|c|}
\hline Model & $\begin{array}{c}\|T / W\| \\
(\%)\end{array}$ & $\begin{array}{c}\left\|E_{m} / W\right\| \\
(\%)\end{array}$ & $\begin{array}{l}B_{0} \\
(\mathrm{G})\end{array}$ \\
\hline ......... & 1.3 & 0 & 0 \\
\hline$\ldots \ldots \ldots$ & 1.3 & $1.1 \times 10^{-8}$ & $10^{8}$ \\
\hline 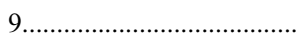 & 1.3 & $1.1 \times 10^{-6}$ & $10^{9}$ \\
\hline 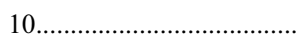 & 1.3 & $1.1 \times 10^{-4}$ & $10^{10}$ \\
\hline 11 & 1.3 & $1.1 \times 10^{-2}$ & $10^{11}$ \\
\hline 12 & 1.3 & 1.1 & $10^{12}$ \\
\hline
\end{tabular}

INPUTS FOR MODELS

NoTes.- $\|T / W\|$ : initial ratio of the rotational energy to the gravitational energy. $\left\|E_{m} / W\right\|$ : initial ratio of the magnetic energy. $B_{0}$ is the strength of the magnetic field in the sphere $\left(r<3.6 \times 10^{9} \mathrm{~cm}\right)$. The digit in the name of each model represents the power index of $B_{0}$.

that is, the average energy of (anti)electron-type neutrinos is used.

Neutrinos are emitted isotropically in the fluid rest frame, so strictly speaking, neutrinos are emitted unisotropically in the coordinate system due to the beaming effect (Rybicki \& Lightman 1979). In fact, the angular frequency is found to become as large as $10^{4} \mathrm{~s}^{-1}$ at the innermost region (Figs. 3 and 10 below), and the rotation velocity, $v_{\phi}$, reaches $\sim 10^{10} \mathrm{~cm} \mathrm{~s}^{-1}$ at most. Thus, the beaming effect may be important, although we did not take into account the effect in this study.

Finally, the models considered in this study are summarized in Table 1 . The digit in the name of each model represents the power index of $B_{0}$.

\section{RESULTS}

We present results of numerical simulations in this section. First of all, we show in Figure 1 the density contour of the central region of the progenitor $\left(r \leq 10^{8} \mathrm{~cm}\right)$ with velocity fields for models 0 and 9 . In the top panels, the density contour with velocity fields for model 0 at $t=2.1 \mathrm{~s}$ (top left panel) and $t=2.2 \mathrm{~s}$ (top right panel) is shown, while in the bottom panels the one for model 9 is shown. The color represents the density $\left(\mathrm{g} \mathrm{cm}^{-3}\right)$ in logarithmic scale $\left(10^{3}-10^{12}\right)$. Vertical axis and horizontal axis represent polar axis (=rotation axis) and equatorial plane, respectively. You can easily find that a jet is launched at $t=2.1 \mathrm{~s}$ for model 9, while no jet occurs for model 0 . In the following subsections we explain the dynamics of these models in detail.

\subsection{Dynamics without Magnetic Fields}

In this subsection we mainly explain the dynamics of model 0 , then that of model 9 is explained in the next subsection. First, we show the accreted energy and mass accretion rate as a function of time in Figure $2 a$. The solid, short-dashed, and dotted lines represent accreted total energy, kinetic energy, and thermal energy $\left(\times 10^{50} \mathrm{ergs}\right)$, respectively, into the black hole. The long-dashed line represents the mass accretion rate $\left(M_{\odot} \mathrm{s}^{-1}\right)$ as a function of time. From this figure, we can understand the following points: (1) The accreted energy amounts to of the order of $10^{52}$ ergs, which is comparable to the explosion energy of a hypernova. Thus, there is a possibility for the models in this study to explain the large explosion energy of a hypernova as long as the released gravitational energy is efficiently converted to the explosion energy. (2) Kinetic energy dominates thermal energy at the inner boundary. This is because almost all thermal energy is extracted in the form of neutrinos, which is seen in Figure $6 a$ below. (3) The mass accretion rate drops almost monotonically from $\sim 10^{-1}$ to $\sim 10^{-3} M_{\odot} \mathrm{s}^{-1}$, which is discussed in $\S \S 3.2$ and 4.2. 


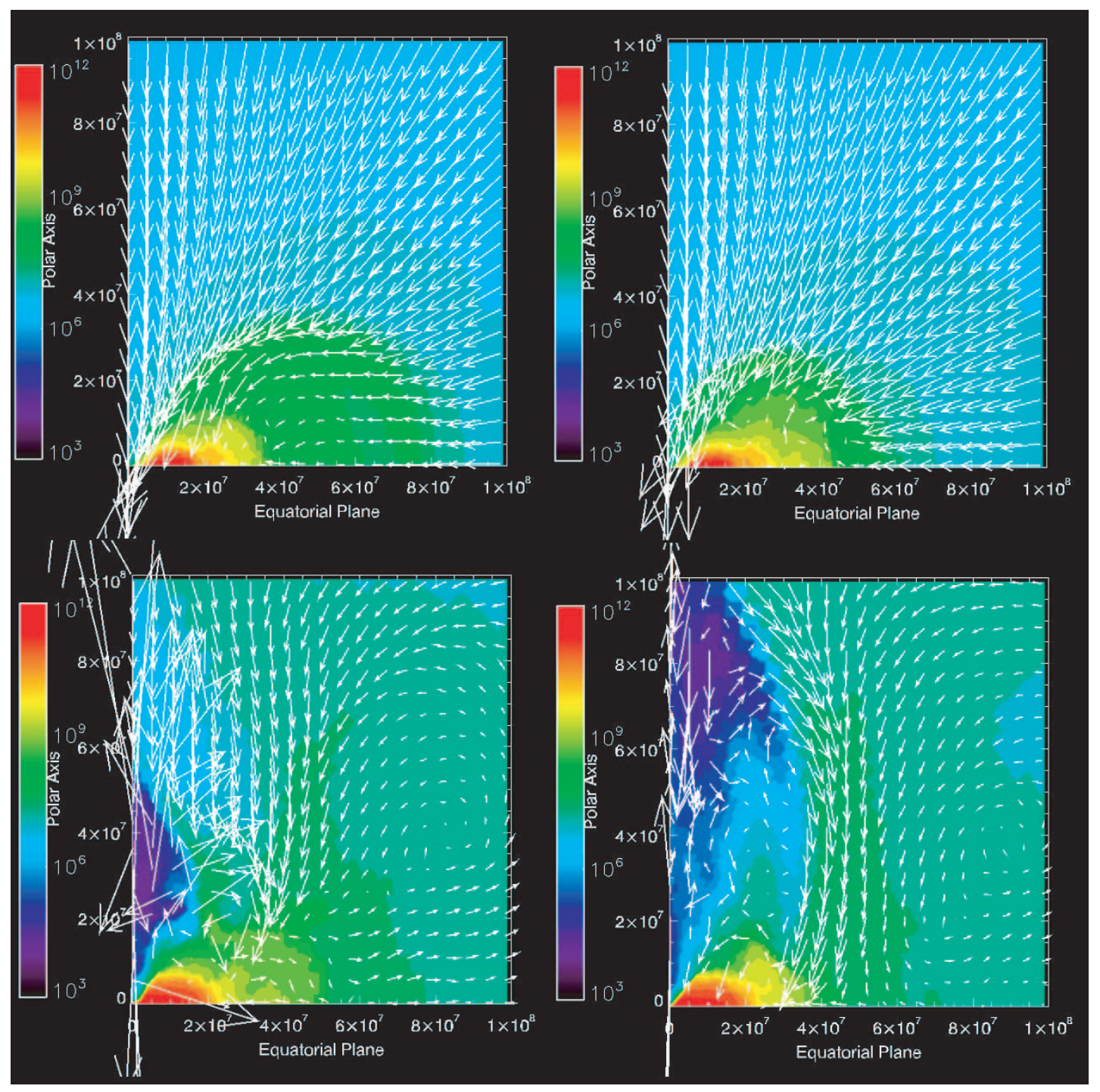

Fig. 1. - Top: Density contour with velocity fields for model 0 at $t=2.1 \mathrm{~s}$ (top left) and $t=2.2 \mathrm{~s}($ top right). Bottom: Same as the top panels, but for model 9 . The color represents the density $\left(\mathrm{g} \mathrm{cm}^{-3}\right)$ in logarithmic scale $\left(10^{3}-10^{12}\right)$. In this figure, the central region of the progenitor $\left(r \leq 10^{8} \mathrm{~cm}\right)$ is shown. Vertical axis and horizontal axis represent rotation axis and equatorial plane, respectively.

Next, we show in Figure 3 profiles of physical quanta of the accretion disk around the equatorial plane. Profiles of density, absolute value of radial velocity, angular frequency, temperature, density scale height, and specific angular momentum in the accretion disk are shown for model 0 at $t=2.2 \mathrm{~s}$. From the figure, we can understand the following points: (1) The density reaches as high as $10^{12} \mathrm{~g} \mathrm{~cm}^{-3}$ around the central region. (2) The inflow velocity $\left(v_{r}\right)$ becomes as low as $10^{6} \mathrm{~cm} \mathrm{~s}^{-1}$ at the central region, by which the mass accretion rate becomes as low as $10^{-3} M_{\odot} \mathrm{s}^{-1}$, as shown in Figure $2 a$ (note that the mass accretion mainly comes from the region between the rotational axis and the disk; see also Igumenshchev \& Abramowicz 2000; Proga \& Begelman 2003).

We show in Figure 4 profiles of the mass fraction for nuclear elements at the equatorial plane for model 0 at $t=2.2 \mathrm{~s}$. The dotdashed, dotted, short-dashed, long-dashed, and solid lines represent the mass fraction of $n, p,{ }^{4} \mathrm{He},{ }^{16} \mathrm{O}$, and ${ }^{56} \mathrm{Ni}$, respectively. From this figure, we can easily see that oxygen is photodissociated into helium at $2 \times 10^{8} \mathrm{~cm}$, while helium is photodissociated into nucleons at $3 \times 10^{7} \mathrm{~cm}$. It is also noted that some ${ }^{56} \mathrm{Ni}$ is seen at $r \leq 3 \times 10^{7} \mathrm{~cm}$ (solid line), which may explain the luminosity of a hypernova as long as it is ejected. This point is discussed in $\S 4.3$. The discontinuity of temperature at $3 \times 10^{7} \mathrm{~cm}$ in
Figure 3 will come from the cooling effect due to the photodisintegration of helium into nucleons.

From now on we show the results on neutrino processes. In Figure 5 we show the contour of the neutrino cooling rate with velocity fields for model 0 at $t=2.2 \mathrm{~s}$. The color represents the emissivity of neutrinos (ergs cm ${ }^{-3} \mathrm{~s}^{-1}$ ) in logarithmic scale $\left(10^{10}-10^{34}\right)$. This emissivity of neutrinos is almost explained by pair captures on free nucleons, as shown in Figure 6 below. We can easily see that the emissivity of neutrinos is high at the region where the accretion disk is formed.

We show the results on neutrino cooling for every neutrino process. We show in Figure $6 a$ the cumulative energy $\left(\times 10^{50} \mathrm{ergs}\right)$ of emitted neutrinos for each process as a function of time for model 0 . The dot-dashed, long-dashed, solid, short-dashed, and dotted lines represent plasmon decay, electron-positron pair annihilation, positron capture, electron capture, and summation of all processes, respectively. It is clearly seen that almost all emitted energy comes from pair captures on free nucleons. Also, the emitted energy amounts to of the order of $10^{52} \mathrm{ergs}$ (strictly speaking, $3.44 \times 10^{52} \mathrm{ergs}$ ), which is comparable to the accreted kinetic energy and much higher than the accreted thermal energy (see Fig. 2a). Thus, we consider almost all thermal energy, which was 


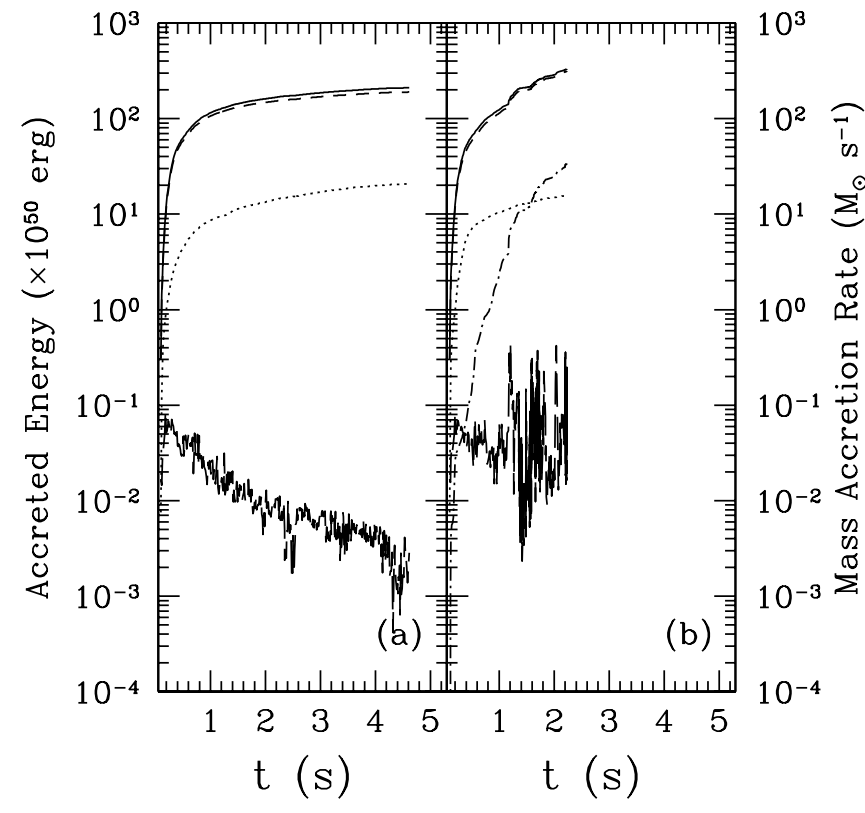

FIG. 2.-Solid, short-dashed, dotted, and dot-dashed lines represent accreted total energy, kinetic energy, thermal energy, and electromagnetic energy $\left(\times 10^{50} \mathrm{ergs}\right)$ into the black hole as a function of time, respectively. Long-dashed lines represent the mass accretion rate $\left(M_{\odot} \mathrm{s}^{-1}\right)$ as a function of time. (a) Case for model 0. (b) Case for model 9. Note that in the case of model 0 , the electromagnetic energy is set to be zero.

comparable to the kinetic energy in the accretion disk, to be extracted by neutrino emission. Note that time evolution of the electron fraction is mainly determined by positron and electron capture processes in the accretion disk.

In Figure 7 we show the contour of the neutrino heating rate with velocity fields for model 0 at $t=2.2 \mathrm{~s}$. The color represents the energy deposition rate $\left(\operatorname{ergs~} \mathrm{cm}^{-3} \mathrm{~s}^{-1}\right)$ in logarithmic scale $\left(10^{10}-10^{34}\right)$. The left panel shows the energy deposition rate due to $\nu_{e}$ and $\bar{\nu}_{e}$ captures on free nucleons, while the right panel shows the energy deposition rate due to $\nu$ and $\bar{\nu}$ pair annihilation. In the pair annihilation, contributions from three flavors are taken into account. It is, of course, that contour of the energy deposition rate due to $\nu_{e}$ and $\bar{\nu}_{e}$ captures that traces the number density of free

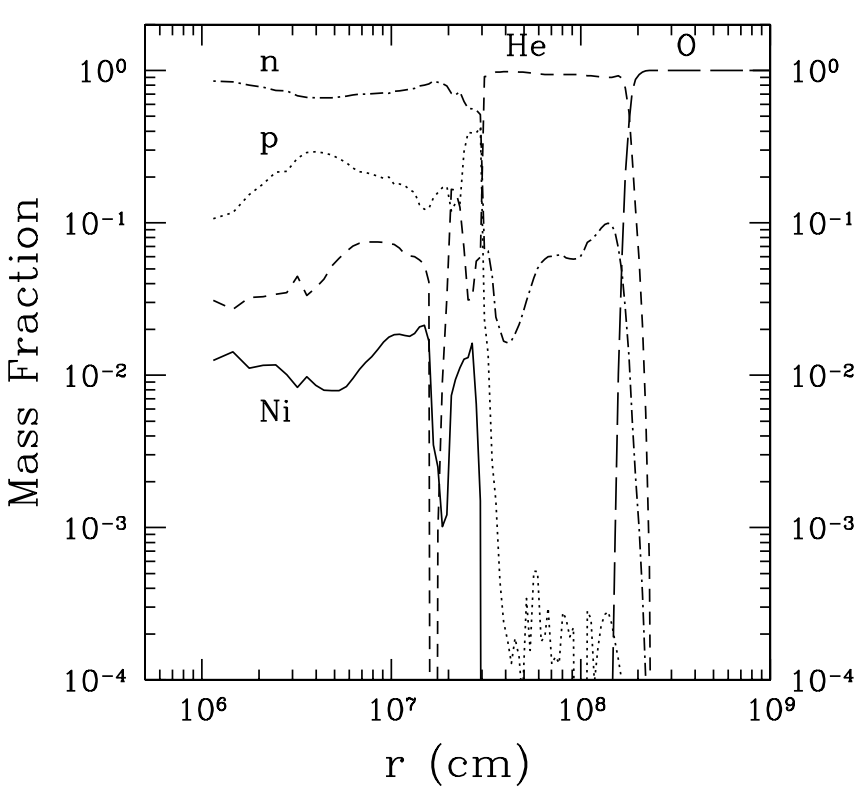

FIG. 4.-Profiles of mass fraction for nuclear elements at the equatorial plane for model 0 at $t=2.2 \mathrm{~s}$. Dot-dashed, dotted, short-dashed, long-dashed, and solid lines represent mass fraction of $n, p,{ }^{4} \mathrm{He},{ }^{16} \mathrm{O}$, and ${ }^{56} \mathrm{Ni}$, respectively.

nucleons, so the energy deposition rate is high around the equatorial plane where the accretion disk is formed. On the other hand, the energy deposition rate due to $\nu$ and $\bar{\nu}$ pair annihilation occurs everywhere, including the region around the polar axis. This feature will be good for launching a jet along the polar axis, as noted by MacFadyen \& Woosley (1999). However, this heating effect is too low to launch a jet in this study (see Fig. 1).

In Figure $8 a$ we show the neutrino luminosity (solid line), energy deposition rate due to $\nu$ and $\bar{\nu}$ pair annihilation (dotted line), and energy deposition rate due to $\nu_{e}$ and $\bar{\nu}_{e}$ captures on free nucleons (dashed line) as a function of time for model 0 . It is clearly seen that the energy deposition rate is much smaller than the neutrino luminosity, which supports our assumption that the system is almost optically thin to neutrinos. Also, we can see that $\nu_{e}$ and $\bar{\nu}_{e}$ captures on free nucleons dominate the $\nu$ and $\bar{\nu}$ pair annihilation process as the heating process. It is also noted that neutrino
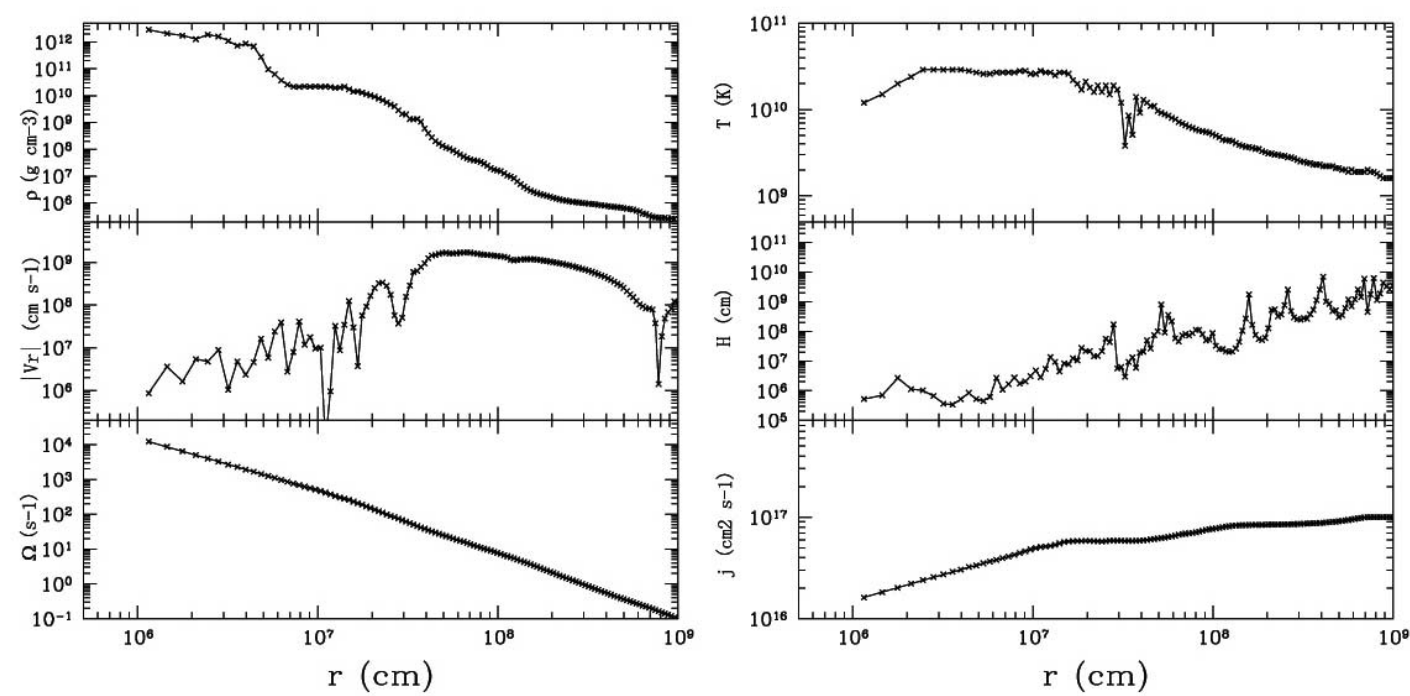

FIG. 3.- Profiles of density, absolute value of radial velocity, angular frequency, temperature, density scale height, and specific angular momentum at the equatorial plane for model 0 at $t=2.2 \mathrm{~s}$. 


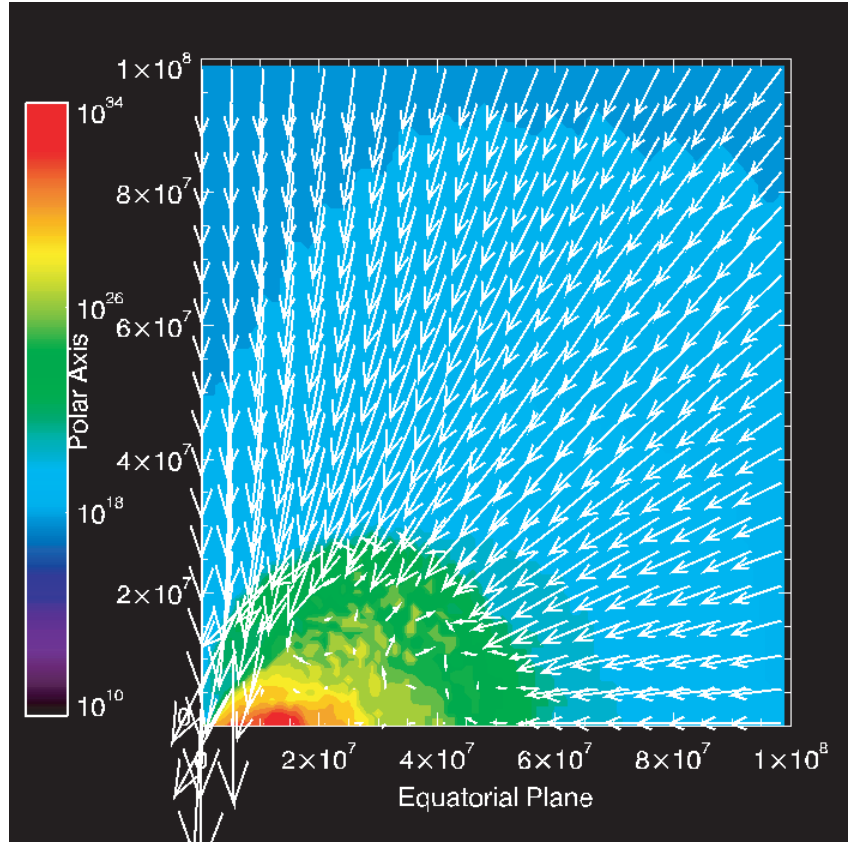

FIG. 5.-Contour of neutrino cooling rate with velocity fields for model 0 at $t=2.2 \mathrm{~s}$. The color represents the emissivity of neutrinos $\left(\mathrm{ergs} \mathrm{cm}^{-3} \mathrm{~s}^{-1}\right)$ in logarithmic scale $\left(10^{10}-10^{34}\right)$.

luminosity and energy deposition rate decrease along with time, which reflects that the mass accretion rate also decreases along with time (Fig. $2 a$ ).

Finally, we show in Figure $9 a$ the integrated deposited energy $\left(\times 10^{50}\right.$ ergs) due to $\nu$ and $\bar{\nu}$ pair annihilation (solid line) and $\nu_{e}$ and $\bar{\nu}_{e}$ captures on free nucleons (dashed line) as a function of time. It is confirmed that $\nu_{e}$ and $\bar{\nu}_{e}$ captures on free nucleons dominate the $\nu$ and $\bar{\nu}$ pair annihilation process as the heating process. However, as shown in Figures 5 and $7, \nu_{e}$ and $\bar{\nu}_{e}$ captures on free nucleons occur mainly in the accretion disk, where the neutrino cooling effect dominates the neutrino heating effect. Thus, $\nu_{e}$ and $\bar{\nu}_{e}$ captures on free nucleons are not considered to work for launching a jet. As for the $\nu$ and $\bar{\nu}$ pair annihilation process, although this process deposits energy everywhere, including the region around the polar axis, the deposited energy amounts to only of the order of $10^{49} \mathrm{ergs}$. This is $10^{-3}$ times smaller than the explosion energy of a hypernova. In fact, as shown in Figure 1, the jet is not launched in model 0 . Thus, we conclude that the efficiency of neutrino heating is too low to launch a jet in this study.

\subsection{Dynamics with Magnetic Fields}

In this subsection we explain the dynamics of model 9 as an example of a collapsar with magnetic fields. Dependence of dynamics on the initial amplitude of magnetic fields is shown in $\S 3.3 .1$.

First, the accreted energy and mass accretion rate as a function of time are shown in Figure $2 b$. The meaning of each line is the same as in Figure $2 a$, although the dot-dashed line, which is not in Figure $2 a$, represents accreted electromagnetic energy $\left(\times 10^{50} \mathrm{ergs}\right)$ into the black hole as a function of time. The reason why the final time of the simulation for model 9 is $2.23 \mathrm{~s}$ is that the amplitude of the magnetic field (in particular, $B_{\phi}$ ) becomes so high at the innermost region that the Alfvén crossing time at the region makes the time step extremely small. Also, it seems that the mass accretion rate does not decrease much in model 9. Rather, it seems to keep $\sim 0.05 M_{\odot} \mathrm{s}^{-1}$. We guess that this is because magnetic fields play a role in transferring the angular momentum from the inner region to the outer region, which makes matter fall

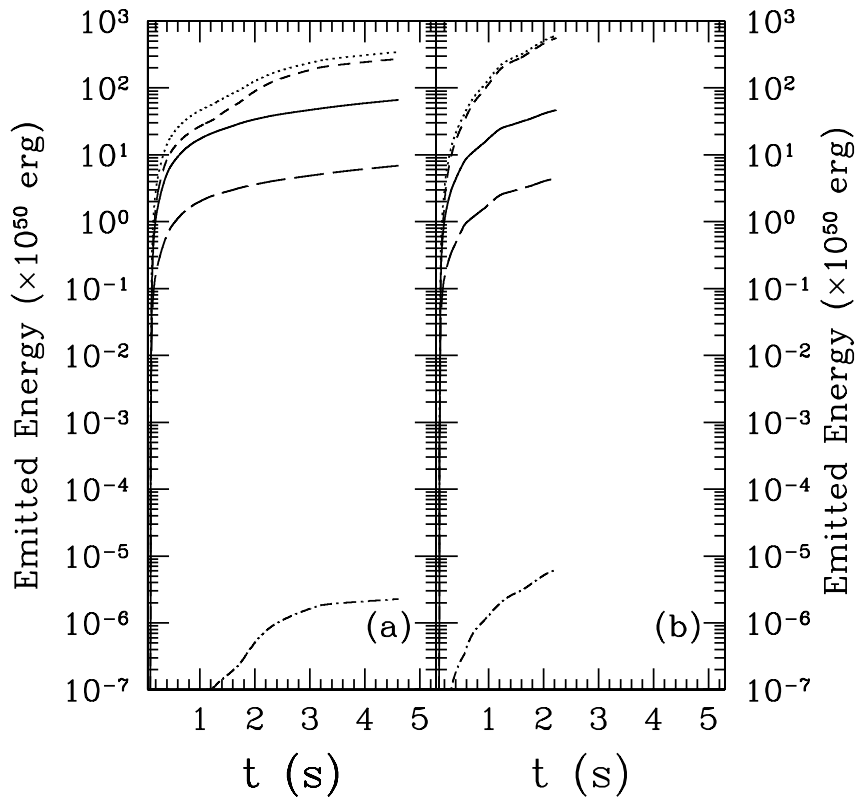

FIG. 6.-Cumulative energy $\left(\times 10^{50} \mathrm{ergs}\right)$ of emitted neutrinos for each process as a function of time. Dot-dashed, long-dashed, solid, short-dashed, and dotted lines represent plasmon decay, electron-positron pair annihilation, positron capture, electron capture, and summation of all processes. (a) Case for model 0. (b) Case for model 9.

into the black hole more efficiently. This point is also discussed with Figures 8-12.

Next, we show in Figure 10 profiles of physical quanta of the accretion disk around the equatorial plane for model 9 at $t=2.2 \mathrm{~s}$. When we compare these profiles with the ones in Figure 3, we can see that the radial velocity is higher in model 9 at small radius. This may reflect that the mass accretion rate is higher in model 9 than model 0 (as stated in $\S 3.1$, the mass accretion mainly comes from the region between the rotational axis and the torus, so we have to note that this feature does not explain the mass accretion rate directly; see also Fujimoto et al. 2006). As for the other profiles, there seems to be no significant difference between the two models.

In Figure 11 we show profiles of amplitude of magnetic fields as a function of radius on the equatorial plane for model 9 at $t=$ $2.2 \mathrm{~s}$. The dotted, solid, and dashed lines represent the amplitude of $B_{r}, B_{\theta}$, and $B_{\phi}$, respectively. It is clearly seen that $B_{\phi}$ dominates within $r=10^{8} \mathrm{~cm}$. Thus, we can conclude that magnetic pressure from $B_{\phi}$ drives the jet along the rotation axis (see Fig. 1). This point is also discussed with Figures 13-15 below. Also, these magnetic fields may play a role in transferring the angular momentum. Since the viscosity parameter $\alpha$ can be estimated as (Balbus \& Hawley 1998; Akiyama et al. 2003)

$$
\alpha \sim \frac{B_{r} B_{\phi}}{4 \pi P},
$$

we plot in Figure 11 the estimated viscosity parameter $\left(\times 10^{-20}\right.$; long-dashed line). This figure suggests that $\alpha$-viscosity coming from the magnetic fields may play a role in transferring the angular momentum at the innermost region effectively. However, the angular momentum cannot be transferred to infinity along the radial direction. This is confirmed by the Alfvén Mach number $\left(\times 10^{-10}\right)$ in the radial direction $\left(\equiv v_{r} / v_{r, \mathrm{~A}} ;\right.$ Fig. 11 , dot-dashed line). At only the innermost region, the flow becomes marginally sub-Alfvénic where the viscous force due to magnetic stress can bring the angular momentum outward. Thus, we believe that the outflow (including the jet) in the polar direction (see Fig. 1, bottom 


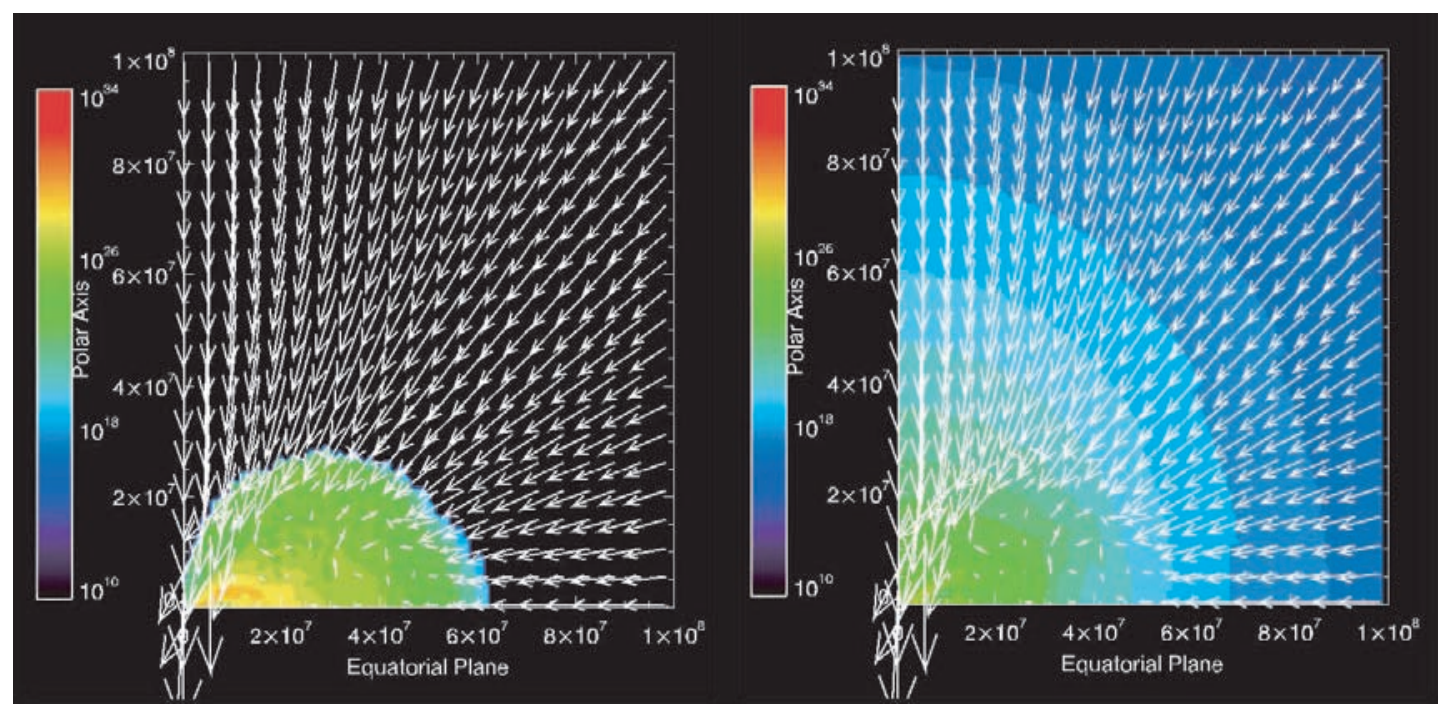

FIG. 7.-Contour of neutrino heating rate with velocity fields for model 0 at $t=2.2 \mathrm{~s}$. The color represents the energy deposition rate (ergs $\mathrm{cm}^{-3} \mathrm{~s}^{-1}$ ) in logarithmic

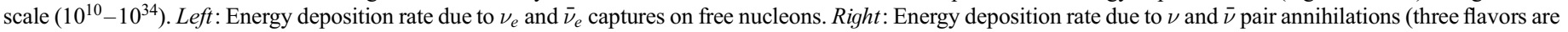
taken into account).

panel) should bring the angular momentum from the innermost region.

As additional information, we found that the velocity of the slow magnetosonic wave is almost the same as the Alfvén velocity. On the other hand, we found that the fluid is subsonic against the fast magnetosonic wave in the simulated region.

We show in Figure 12 the profiles of specific angular momentum $\left(\mathrm{cm}^{2} \mathrm{~s}^{-1}\right)$ on the equatorial plane for model 0 (dotted line) and model 9 (short-dashed line) at $t=2.2 \mathrm{~s}$. The profiles of angular momentum density $\left(\mathrm{g} \mathrm{cm}^{-1} \mathrm{~s}^{-1}\right)$ for model 0 (longdashed line) and model 9 (solid line) are also shown in the figure. We confirmed that the specific angular momentum is not so different between model 0 and model 9 , but it is found that the angular momentum density is lower in model 9 compared with model 0 at the inner region. This feature might reflect that the

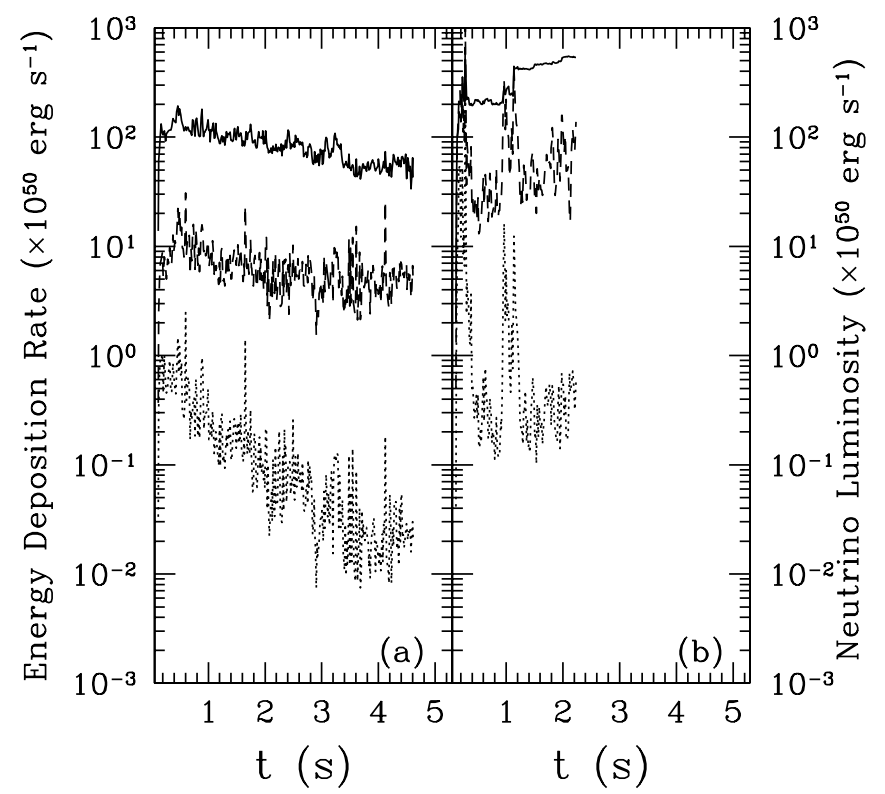

FIG. 8.-Neutrino luminosity (solid lines), energy deposition rate due to $\nu$ and $\bar{\nu}$ pair annihilations (dotted lines), and energy deposition rate due to $\nu_{e}$ and $\bar{\nu}_{e}$ captures on free nucleons (dashed line) as a function of time. The units are $10^{50} \mathrm{ergs} \mathrm{s}^{-1}$. (a) Case for model 0. (b) Case for model 9. matter falls into the black hole efficiently in model 9 at small radius (see Fig. 11). This picture seems to be consistent with the almost constant accretion rate in model 9 (Fig. $2 b$ ).

The cumulative energy $\left(\times 10^{50} \mathrm{ergs}\right)$ of emitted neutrinos for each process as a function of time for model 9 is shown in Figure $6 b$; neutrino luminosity, energy deposition rate due to $\nu$ and $\bar{\nu}$ pair annihilation, and energy deposition rate due to $\nu_{e}$ and $\bar{\nu}_{e}$ captures on free nucleons as a function of time for model 9 are shown in Figure $8 b$; and integrated deposited energy due to $\nu$ and $\bar{\nu}$ pair annihilation and $\nu_{e}$ and $\bar{\nu}_{e}$ captures on free nucleons as a function of time for model 9 are shown in Figure $9 b$. The meaning of each line in each figure is same as the one used for model 0 . We can derive a similar conclusion for the role of the neutrino heating effect in model 9 as in model 0 . As for the $\nu_{e}$ and $\bar{\nu}_{e}$ captures on free nucleons, the cumulative deposited energy becomes as high

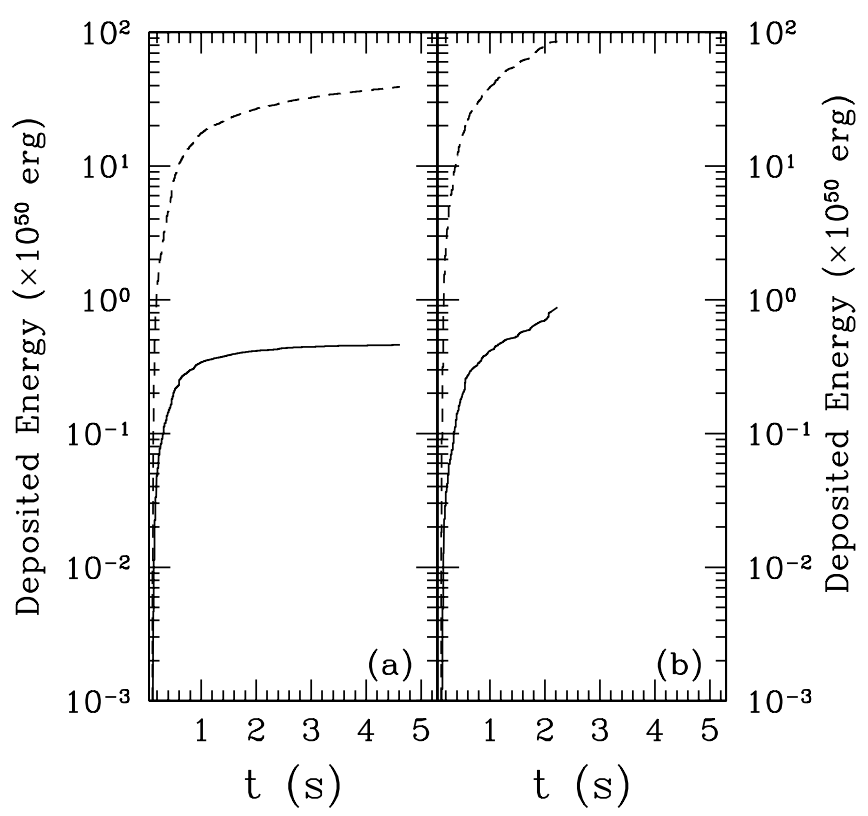

FIG. 9.- Integrated deposited energy $\left(\times 10^{50} \mathrm{ergs}\right)$ due to $\nu$ and $\bar{\nu}$ pair annihilations (solid lines) and $\nu_{e}$ and $\bar{\nu}_{e}$ captures on free nucleons (dashed lines) as a function of time. (a) Case for model 0. (b) Case for model 9. 

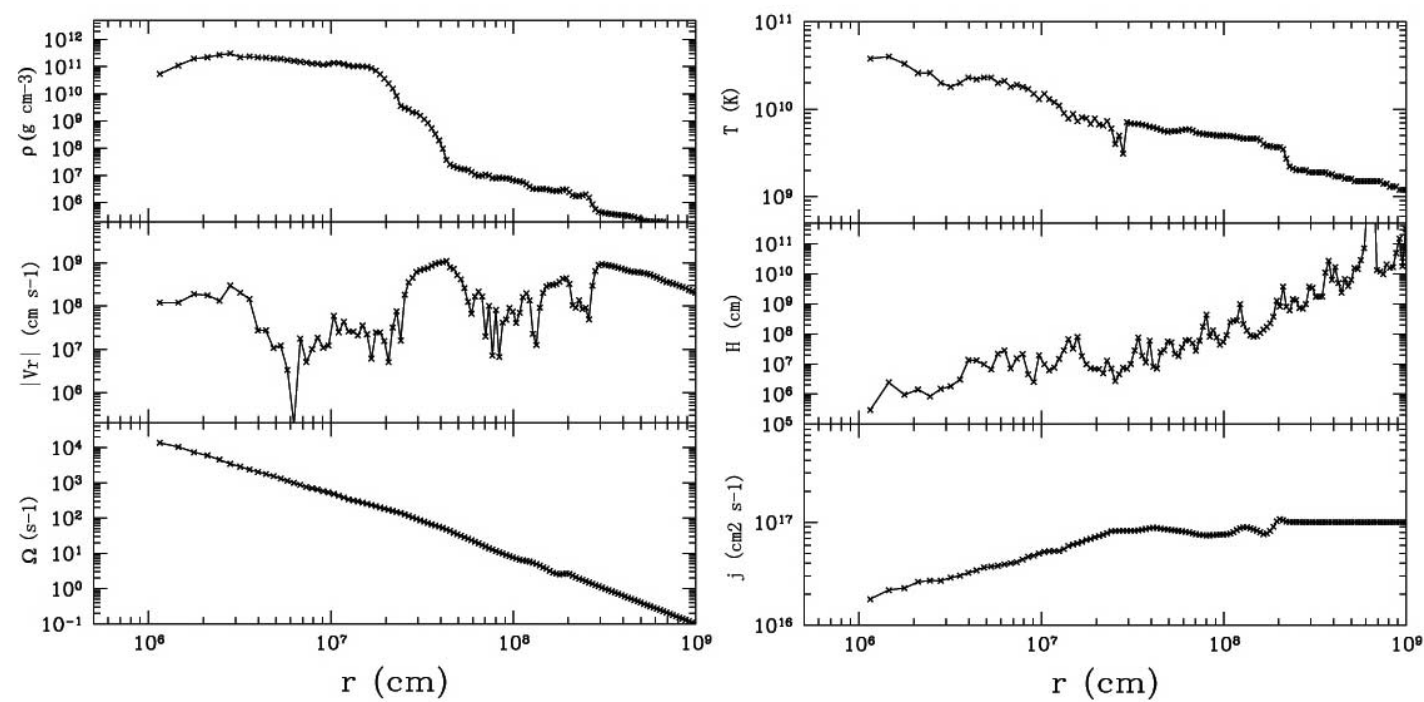

FIG. 10.- Same as Fig. 3, but for model 9. Note that the radial velocity profile is not similar to model 0 at small radius.

as $10^{52} \mathrm{ergs}$, which is comparable to the explosion energy of a hypernova. However, this heating process occurs mainly in the accretion disk, where the neutrino cooling effect dominates the neutrino heating effect. Thus, $\nu_{e}$ and $\bar{\nu}_{e}$ captures on free nucleons are not considered to work for launching a jet. As for the $\nu$ and $\bar{\nu}$ pair annihilation process, the deposited energy amounts to no more than $10^{50}$ ergs. Thus, we conclude that the neutrino heating effect in model 9 is too inefficient to launch a GRB jet and cause a hypernova. It has to be noted that the energy deposition rate due to pair captures on free nucleons sometimes becomes larger than the neutrino luminosity in Figure $8 b$. This means that the optically thin limit breaks down at that time. This point is also discussed in $\S 4.1$. We also found that the neutrino luminosity, energy de-

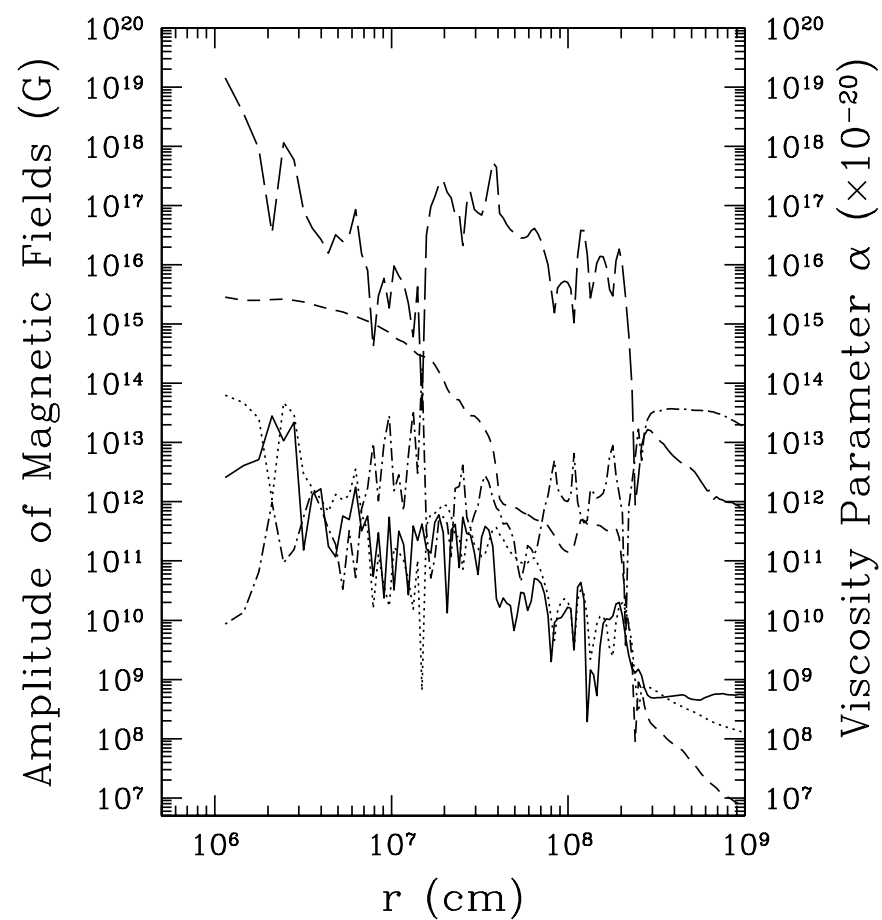

FIG. 11.-Profile of amplitude of magnetic fields and estimated viscosity parameter $\alpha\left(\times 10^{-20}\right)$ on the equatorial plane for model 9 at $t=2.2 \mathrm{~s}$. Dotted, solid, and short-dashed lines represent the amplitude of $B_{r}, B_{\theta}$, and $B_{\phi}$, respectively, while the long-dashed line represents the estimated viscosity parameter. The dotdashed line represents the Alfvén Mach number $\left(\times 10^{-10}\right)$ in the radial direction. position rate, and integrated deposited energy seem to be higher in model 9 than in model 0 . We believe that this feature comes from the high mass accretion rate (high rate of release of gravitational energy) caused by angular momentum transfer due to magnetic fields.

We show in Figure 13 time evolution of energy of magnetic fields $\left(\times 10^{50} \mathrm{ergs}\right)$ in the whole calculated region for the case of model 9 . The dotted, solid, and dashed lines represent energy in the form of $B_{r}, B_{\theta}$, and $B_{\phi}$, respectively. We can see that $B_{\phi}$ grows rapidly and dominates other components, although $B_{\phi}$ is much smaller than other components at first (note that the one-dimensional simulation of the spherical collapse of the progenitor is done until $t=0.1 \mathrm{~s}$ [see $\S 2.2$ ], so $B_{\phi}$ is set to be 0 until $t=0.1 \mathrm{~s}$ ). $B_{\phi}$ is amplified by the winding effect and launches a jet along the polar

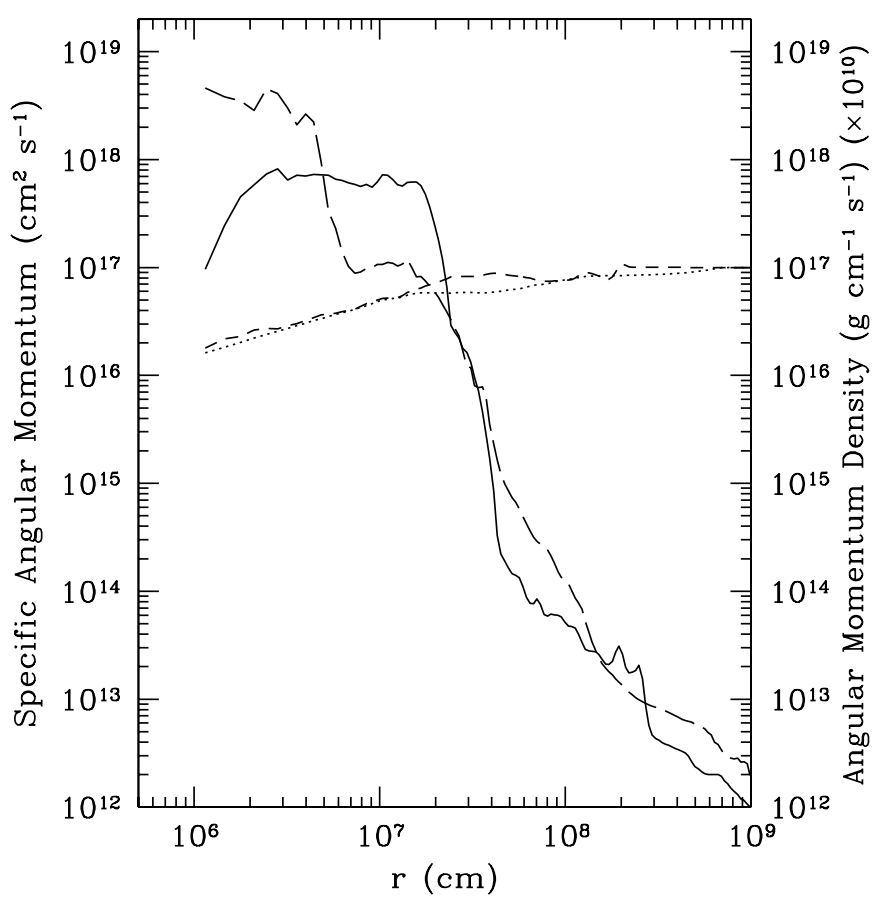

FIG. 12.-Profiles of angular momentum density $\left(\mathrm{g} \mathrm{cm}^{-1} \mathrm{~s}^{-1}\right)$ for model 0 (long-dashed line) and model 9 (solid line) on the equatorial plane at $t=2.2 \mathrm{~s}$. Profiles of specific angular momentum $\left(\mathrm{cm}^{2} \mathrm{~s}^{-1}\right)$ are also shown for model 0 (dotted line) and model 9 (short-dashed line). 


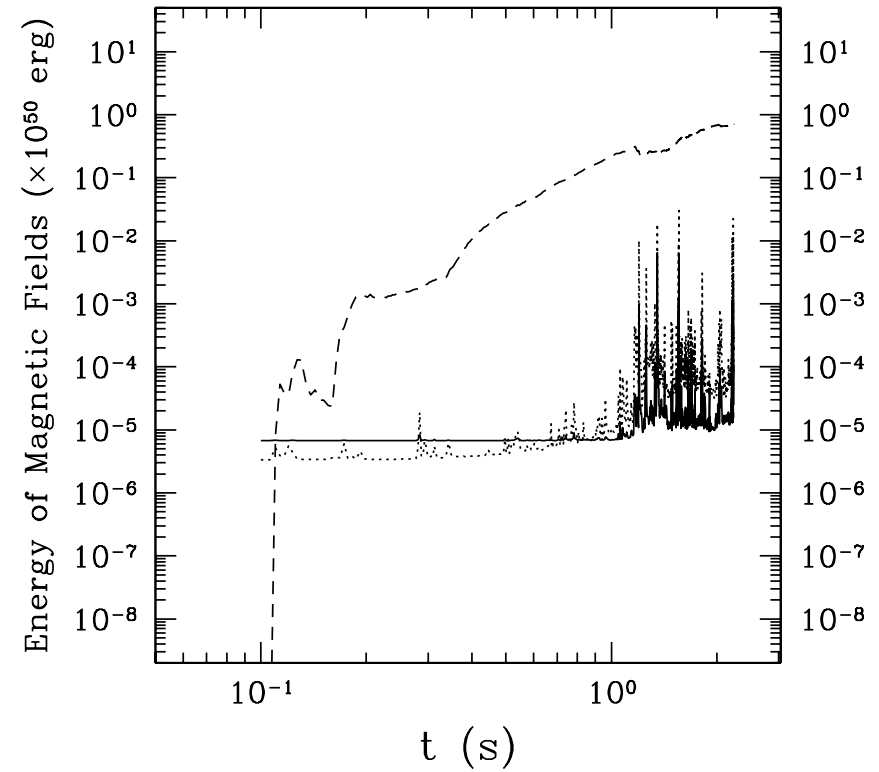

FIG. 13.-Evolution of total energy of magnetic fields $\left(\times 10^{50} \mathrm{ergs}\right)$ for the case of model 9. Dotted, solid, and dashed lines represent energy in the form of $B_{r}, B_{\theta}$, and $B_{\phi}$, respectively. Note that a one-dimensional simulation of the spherical collapse of the progenitor is done until $t=0.1 \mathrm{~s}$ (see $\S 2.2)$, so $B_{\phi}$ is set to be 0 until $t=0.1 \mathrm{~s}$.

axis (see Fig. 1). The total energy of $B_{\phi}$ at the final stage of the simulation in this study is of the order of $10^{50} \mathrm{ergs}$, which is much smaller than the explosion energy of a hypernova. However, the total energy of $B_{\phi}$ is increasing almost monotonically. As mentioned above, the final time of the simulation for model 9 is determined due to the reason that the Alfvén crossing time becomes extremely small at the innermost region. When we can overcome this problem and simulate model 9 further, there is a possibility that the energy of $B_{\phi}$ becomes comparable to the explosion energy of a hypernova. The evolution of $B_{r}$ and $B_{\theta}$ is similar to the one by magnetic rotational instability (MRI), which is discussed in $\S 4.2$.

We show in Figure 14 the contour of plasma beta $\left(=p_{\text {gas }+ \text { radiation }} /\right.$ $\left.p_{\text {mag }}\right)$ with magnetic fields $\left(B_{r}\right.$ and $\left.B_{\theta}\right)$ for model 9 at $t=2.2 \mathrm{~s}$. In $p_{\text {gas }}+$ radiation, the degenerated pressure of electrons is included. The color represents the plasma beta in logarithmic scale $\left(10^{-1}\right.$ to $10^{8}$ ). The minimum value of plasma beta in this region is 0.193 . We can see clearly that the beta value is low in the jet region, from which we can understand that the jet is launched by the magnetic fields (in particular, $B_{\phi}$ ).

Finally, we have performed these simulations presented in $\S \S 3.1$ and 3.2 without calculating self-gravity and found that the dynamics of the collapsar is not changed much.

\subsection{Toward Discussions}

Before we discuss the results mentioned above, we present two more results in this section. One is the dependence of dynamics on initial amplitude of magnetic fields, and the other is the explosive nucleosynthesis.

\subsubsection{Dependence on Initial Amplitude of Magnetic Fields and on Resolution of Grids}

Here we show the dependence of dynamics on initial amplitude of magnetic fields. In Figure 15, evolutions of total energy of magnetic fields $\left(\times 10^{50}\right.$ ergs) for model 8 (top left panel), model 10 (top right panel), model 11 (bottom left panel), and model 12 (bottom right panel) are shown. The final time of the simulation is determined, like in model 9 , by the reason that the Alfvén

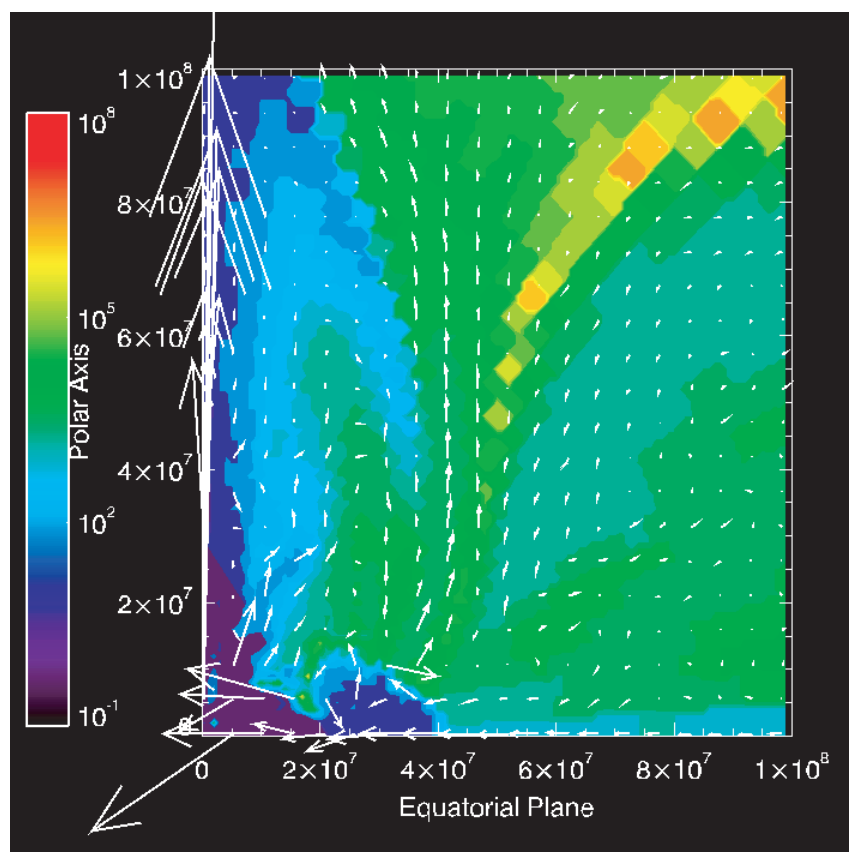

FIG. 14. - Contour of plasma beta $\left(=p_{\text {gas }+ \text { radiation }} / p_{\text {mag }}\right)$ with magnetic fields $\left(B_{r}\right.$ and $B_{\theta}$ ) for model 9 at $t=2.2 \mathrm{~s}$. The color represents the plasma beta in logarithmic scale $\left(10^{-1}\right.$ to $\left.10^{8}\right)$.

crossing time becomes extremely small at the innermost region. In all models, the energy of $B_{\phi}$ amounts to $10^{49}-10^{50} \mathrm{ergs}$, which means that the amplitude of $B_{\phi}$ becomes as strong as $10^{15} \mathrm{G}$. This can be understood by simple calculations: the typical radius of the innermost region is of the order of $10^{6} \mathrm{~cm}$, so the volume of the region times the energy density of magnetic fields becomes $E_{B}=8.3 \times 10^{49}\left(B / 2 \times 10^{15} \mathrm{G}\right)^{2}\left(r / 5 \times 10^{6} \mathrm{~cm}\right)^{3}$ ergs.

The total energy of $B_{r}$ and $B_{\theta}$ components does not change much with time. In particular, in models 10-12, their total energy hardly changes. When we compare $B_{r}$ with $B_{\theta}, B_{r}$ seems to be more unstable than $B_{\theta}$ (see also Fig. 13). This is similar to the results of the local simulations of MRI, which is discussed in $\S 4.2$.

We summarize the features of the launched jet at the final stage of simulations for every model in Table 2 . We define the jet

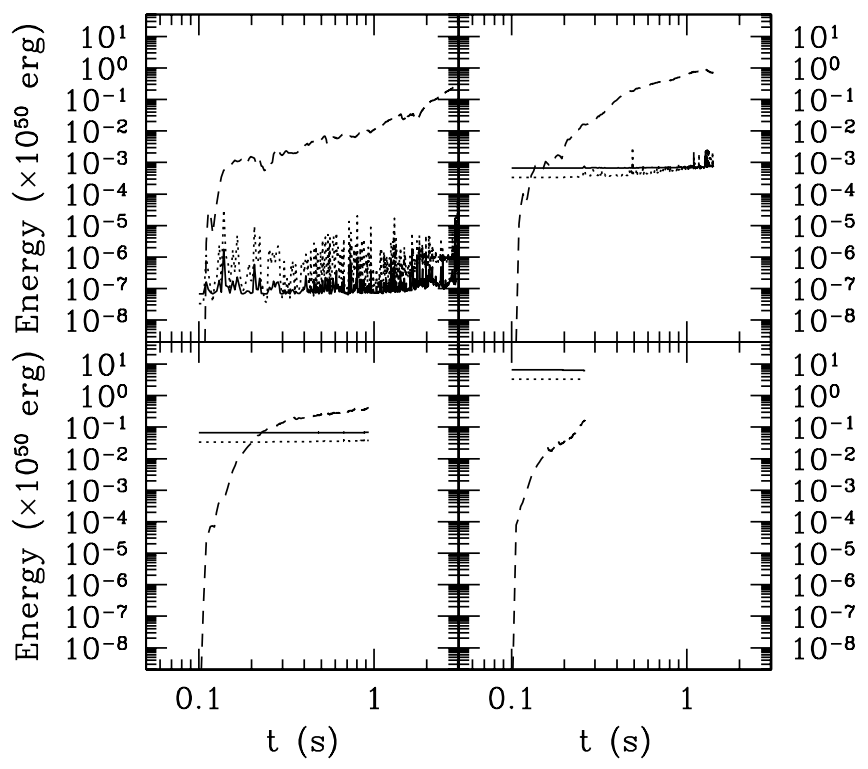

FIG. 15.-Same as Fig. 13, but for model 8 (top left), model 10 (top right), model 11 (bottom left), and model 12 (bottom right). 
TABLE 2

OUtPuts FOR MODEls

\begin{tabular}{|c|c|c|c|c|c|}
\hline Model & $\begin{array}{c}\theta_{J} \\
(\mathrm{deg})\end{array}$ & $\begin{array}{c}E_{J} \\
(\mathrm{ergs})\end{array}$ & $\begin{array}{c}M_{J} \\
\left(M_{\odot}\right)\end{array}$ & $\Gamma^{f}-1$ & $E_{J}^{\mathrm{mag}} / E_{J}$ \\
\hline \multirow[t]{3}{*}{$8 \ldots \ldots \ldots \ldots \ldots$} & 5 & $6.97 \mathrm{E}+47$ & $4.88 \mathrm{E}-6$ & $7.98 \mathrm{E}-2$ & $4.75 \mathrm{E}-2$ \\
\hline & 10 & $7.40 \mathrm{E}+47$ & $5.39 \mathrm{E}-6$ & $7.67 \mathrm{E}-2$ & $5.36 \mathrm{E}-2$ \\
\hline & 15 & $7.66 \mathrm{E}+47$ & $5.56 \mathrm{E}-6$ & $7.70 \mathrm{E}-2$ & $6.12 \mathrm{E}-2$ \\
\hline \multirow[t]{3}{*}{$9 \ldots \ldots \ldots \ldots \ldots \ldots$} & 5 & $2.96 \mathrm{E}+47$ & $2.42 \mathrm{E}-7$ & $6.81 \mathrm{E}-1$ & $4.71 \mathrm{E}-2$ \\
\hline & 10 & $4.27 \mathrm{E}+47$ & $3.13 \mathrm{E}-7$ & $7.60 \mathrm{E}-1$ & $3.60 \mathrm{E}-1$ \\
\hline & 15 & $4.87 \mathrm{E}+47$ & $4.38 \mathrm{E}-7$ & $6.21 \mathrm{E}-1$ & $3.45 \mathrm{E}-2$ \\
\hline \multirow[t]{3}{*}{$10 \ldots \ldots \ldots \ldots \ldots$} & 5 & $8.17 \mathrm{E}+45$ & $1.97 \mathrm{E}-8$ & $2.32 \mathrm{E}-1$ & $2.83 \mathrm{E}-1$ \\
\hline & 10 & $9.08 \mathrm{E}+45$ & $2.12 \mathrm{E}-8$ & $2.39 \mathrm{E}-1$ & $2.72 \mathrm{E}-1$ \\
\hline & 15 & $1.32 \mathrm{E}+46$ & $3.65 \mathrm{E}-8$ & $2.03 \mathrm{E}-1$ & $2.13 \mathrm{E}-1$ \\
\hline \multirow[t]{3}{*}{$11 \ldots \ldots \ldots \ldots \ldots$} & 5 & $1.05 \mathrm{E}+48$ & $1.16 \mathrm{E}-5$ & $5.05 \mathrm{E}-2$ & $2.74 \mathrm{E}-1$ \\
\hline & 10 & $1.16 \mathrm{E}+48$ & $1.24 \mathrm{E}-5$ & $5.22 \mathrm{E}-2$ & $2.56 \mathrm{E}-1$ \\
\hline & 15 & $1.32 \mathrm{E}+48$ & $1.36 \mathrm{E}-5$ & $5.44 \mathrm{E}-2$ & $2.33 \mathrm{E}-1$ \\
\hline \multirow[t]{3}{*}{$12 \ldots \ldots \ldots \ldots \ldots$} & 5 & $1.45 \mathrm{E}+49$ & $1.16 \mathrm{E}-4$ & $6.99 \mathrm{E}-2$ & $1.69 \mathrm{E}-1$ \\
\hline & 10 & $1.77 \mathrm{E}+49$ & $1.52 \mathrm{E}-4$ & $6.50 \mathrm{E}-2$ & $1.57 \mathrm{E}-1$ \\
\hline & 15 & $2.39 \mathrm{E}+49$ & $2.47 \mathrm{E}-4$ & $5.42 \mathrm{E}-2$ & $1.29 \mathrm{E}-1$ \\
\hline
\end{tabular}

Notes. $-E_{J}, M_{J}, \Gamma^{f}$, and $E_{J}^{\mathrm{mag}} / E_{J}$ are mass, total energy, terminal bulk Lorentz factor, and ratio of the magnetic energy relative to total energy of the jet at the final stage of the simulations, respectively. The quantity $\theta_{J}$ is the assumed opening angle of the jet. See $\S 3.3 .1$ for details.

as follows: (1) the region within some opening angle $\left(\theta_{J}\right)$; $(2)$ the region where total energy (i.e., summation of kinetic, thermal, electromagnetic, and gravitational energies) is positive at the final stage of the simulations; (3) the amplitude of the velocity is larger than $5 \times 10^{9} \mathrm{~cm} \mathrm{~s}^{-1}$. In Table 2, total energy, mass, terminal bulk Lorentz factor, and ratio of the magnetic energy relative to total energy of the jet are shown assuming that $\theta_{J}=5^{\circ}, 10^{\circ}$, and $15^{\circ}$ for each model. The terminal bulk Lorentz factor is estimated by assuming that total energy goes into kinetic energy during expansion.

We can see that the terminal bulk Lorentz factor in every model is much smaller than the required value $(\sim 300)$ for GRB jets. Also, the energy of the jet in every model is much smaller than the typical energy of a GRB. Thus, we conclude that the jets seen in this study will not be GRB jets.

Also, we show in the left panel of Figure 16 the evolution of total energy of magnetic fields for the case of model 9 (same

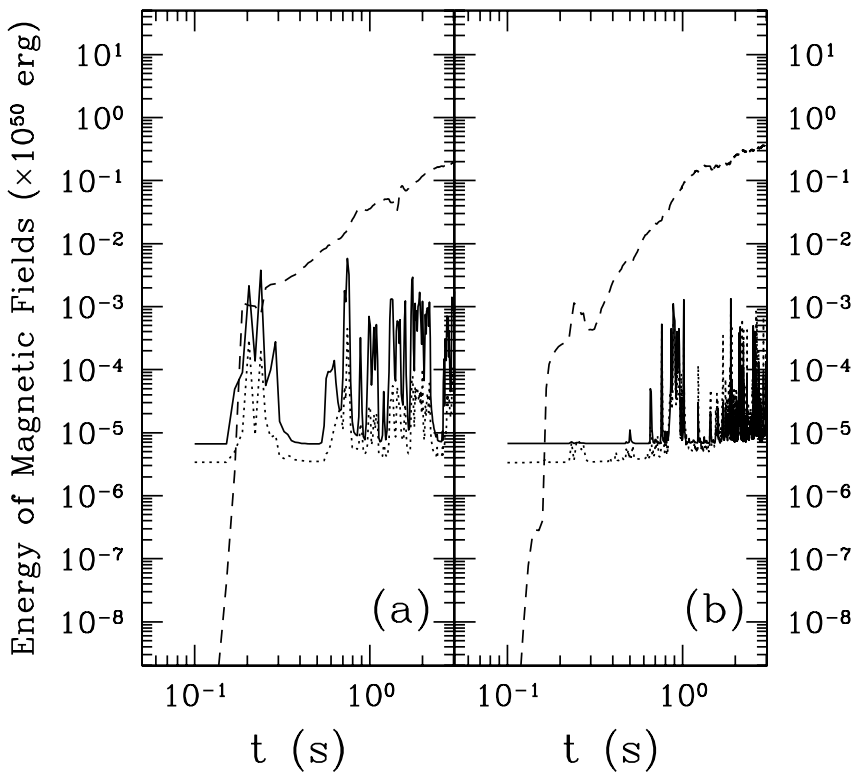

TABLE 3

ABUndance of ${ }^{56} \mathrm{Ni}$

\begin{tabular}{cll}
\hline \multirow{2}{*}{ Model } & $\begin{array}{l}M_{\mathrm{Ni}}^{\mathrm{esc}} \\
\left(M_{\odot}\right)\end{array}$ & $\begin{array}{l}M_{\mathrm{Ni}}^{\text {tot }} \\
\left(M_{\odot}\right)\end{array}$ \\
\hline & $6.04 \mathrm{E}-10$ & $3.79 \mathrm{E}-3$ \\
& $7.52 \mathrm{E}-11$ & $5.92 \mathrm{E}-3$ \\
& $1.09 \mathrm{E}-7$ & $3.95 \mathrm{E}-3$ \\
& $1.40 \mathrm{E}-6$ & $1.61 \mathrm{E}-3$ \\
& $5.62 \mathrm{E}-7$ & $1.12 \mathrm{E}-3$ \\
\hline
\end{tabular}

Notes. $-M_{\mathrm{Ni}}^{\text {esc }}$ and $M_{\mathrm{Ni}}^{\text {tot }}$ represent the mass of ${ }^{56} \mathrm{Ni}$ in the regions where total energy (i.e., summation of kinetic energy, thermal energy, and gravitational energy) is positive and the total mass of ${ }^{56} \mathrm{Ni}$ in the whole simulated region at the final stage of the simulations, respectively.

with Fig. 13), but for $150(r) \times 5(\theta)$ grid points (left panel) and $150(r) \times 20(\theta)$ grid points (right panel) to show the dependence of results on the grid resolution. It is found that the growth rate of $B_{r}$ and $B_{\theta}$ components depends on the grid resolution. On the other hand, the growth rate of $B_{\phi}$ does not depend on the grid resolution (that is, the energy in $B_{\phi}$ fields becomes $10^{49}-10^{50} \mathrm{ergs}$ in $1-2 \mathrm{~s}$ ). The saturation level of $B_{r}$ and $B_{\theta}$ does not depend much on the grid resolution. This is discussed in $\S 4.2$. We have also done a simulation of model 9 with finer resolution $[300(r) \times 60(\theta)]$, although neutrino-antineutrino pair annihilation is not included to save CPU time. The simulation region is set to be $\left(10^{6} \mathrm{~cm} \leq\right.$ $r \leq 10^{9} \mathrm{~cm}, 0^{\circ} \leq \theta \leq 90^{\circ}$ ). The minimum radial grid is set to be $3 \times 10^{5} \mathrm{~cm}$. We have found that the dynamics of collapsars is hardly changed. We have found that an accretion disk is formed around the black hole and a jet is launched at $t=1.98 \mathrm{~s}$. Moreover, we have found that the evolution of magnetic fields is hardly changed, which is shown in the right panel of Figure 16. This result means that a jet is driven by magnetic fields and the standard resolution of our study $[150(r) \times 30(\theta)]$ is not so bad.

\subsubsection{Nucleosynthesis}

In this section we present results on explosive nucleosynthesis. We show in Table 3 the mass of ${ }^{56} \mathrm{Ni}$ in the regions where total energy is positive $\left(M_{\mathrm{Ni}}^{\text {esc }}\right)$ and the total mass of ${ }^{56} \mathrm{Ni}$ in the whole simulated region $\left(M_{\mathrm{Ni}}^{\text {tot }}\right)$ for each model. These amounts

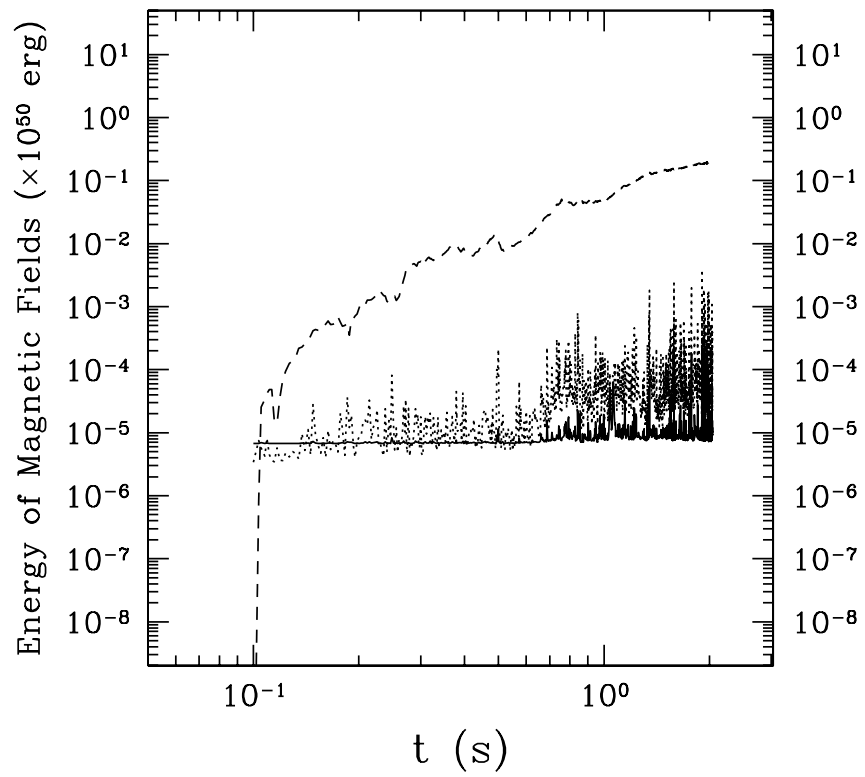

FIG. 16. - Left: Same as Fig. 13, but for $(a) 150(r) \times 5(\theta)$ grid points and $(b) 150(r) \times 20(\theta)$ grid points. Right: Same as Fig. 13 , but for $300(r) \times 60(\theta)$ grid points. In this simulation, the neutrino-antineutrino pair annihilation effect is not included to save CPU time. The simulation region is $\left(10^{6} \mathrm{~cm} \leq r \leq 10^{9} \mathrm{~cm}, 0^{\circ} \leq \theta \leq 90^{\circ}\right)$. 


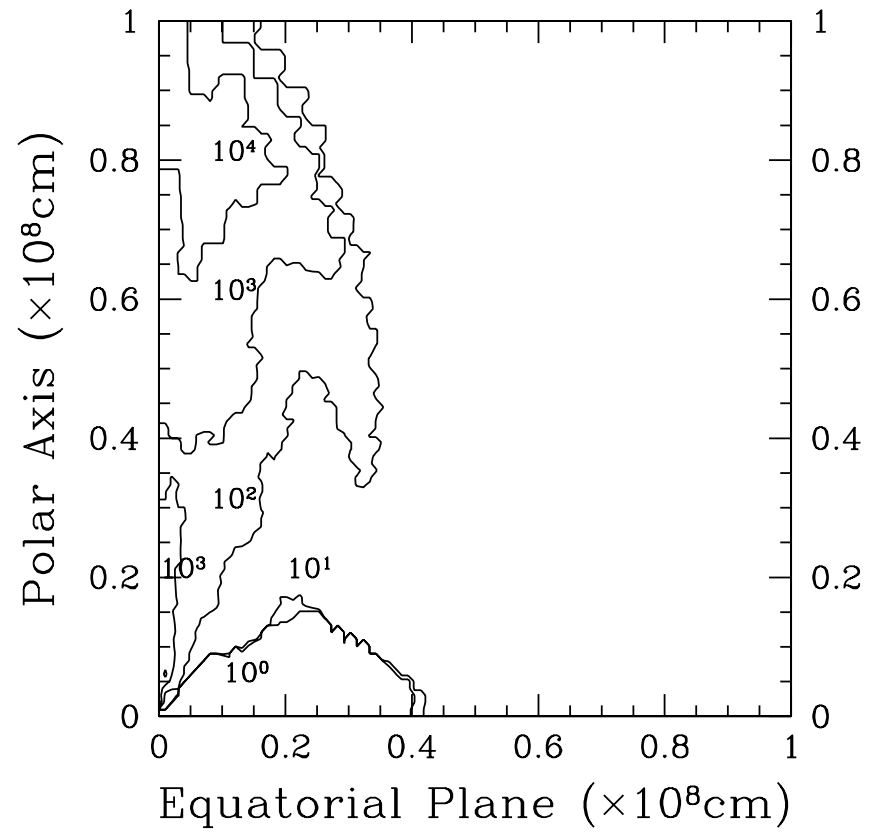

FIG. 17.-Contour of entropy per baryon in units of $k_{b}$ at $t=2.2 \mathrm{~s}$ for model 9 . The range of the contour is from 1 to $10^{5}$

are estimated at the final stage of the simulations. The ejected mass of ${ }^{56} \mathrm{Ni}, M_{\mathrm{Ni}}^{\mathrm{esc}}$, is too little to explain the luminosity of a hypernova, although a considerable amount of ${ }^{56} \mathrm{Ni}$ is synthesized in the accretion disk $\left(M_{\mathrm{Ni}}^{\mathrm{tot}}\right)$. We found that the ejected mass is mainly composed of $n, p$, and He. This is supported by Figure 17, where the entropy per baryon at $t=2.2 \mathrm{~s}$ for model 9 is shown in units of $k_{b}$. In the jet region, the entropy per baryon is remarkably high, so it is natural that light elements dominate in the jet region.

Finally, we show in Figure 18 the contour of electron fraction $\left(Y_{e}\right)$ with velocity fields for model 0 at $t=2.2 \mathrm{~s}$ (left panel) and model 9 at $t=2.2 \mathrm{~s}$ (right panel). The color represents the electron fraction on a linear scale $(0.1-0.540)$. We can easily see that $Y_{e}$ becomes low in the accretion disk. This is because electrons are degenerated and electron capture dominates positron capture at this region. Also, we can see that the mass element with low $Y_{e}$ is ejected in model 9. Also, the mass element with high $Y_{e}$ (highest value is 0.522 ) is also ejected in model 9 from the inside of the low $-Y_{e}$ jet along the polar axis near the black hole.

Since entropy per baryon is very high in the jet region (Fig. 17), these mass elements may cause $r$-process and/or $r / p$-process nucleosynthesis. Here we have to comment on the electron fraction at the high-density region. The value of the electron fraction is solved of the order of 0.1 in the accretion disk. This value is obtained assuming that the chemical potential of electron-type neutrinos is zero. If careful treatment of neutrino transfer is done, the chemical potential of electron-type neutrinos may prohibit the electron fraction from being as low as 0.1 . This point is discussed in $\S \S 4.1$ and 4.3 .

\section{DISCUSSIONS}

In this section we discuss our numerical results and prospect for future works. We discuss neutrino physics, effects of magnetic fields, nucleosynthesis, general relativistic effects, initial conditions, and prospects for improvements of our numerical code.

\subsection{Neutrino Physics}

In this study we found that deposited energy due to neutrino pair annihilation is too small to explain the explosion energy of a hypernova and a GRB (Fig. 9). Even though the deposited energy by electron-type neutrino capture on free nucleons can be comparable to the explosion energy of a hypernova in model 9 (Fig. 9), the deposition region is the high-density region (Fig. 7) where the cooling effect dominates the heating effect (Fig. 5). In particular, no jet was found in the numerical simulations of model 0 (Fig. 1). The energetics of this system can be understood from Figures 2, 6, and 9. The released gravitational energy by collapse is the energy source of accreted energy and neutrinos. From Figures 2 and 6, we can understand that the kinetic energy and thermal energy share the released gravitational energy almost equally, and then almost thermal energy was extracted in the form of neutrinos. The total energies of accreted energy and neutrinos are of the order of $10^{52} \mathrm{ergs}$, and then the emitted neutrino energy was deposited into matter through weak interactions. Its efficiency is less than $1 \%$ for neutrino pair annihilation, as can be seen in Figures 6 and 9. As for the efficiency of electron-type neutrino capture, it amounts to $\sim 10 \%-20 \%$. This means that the

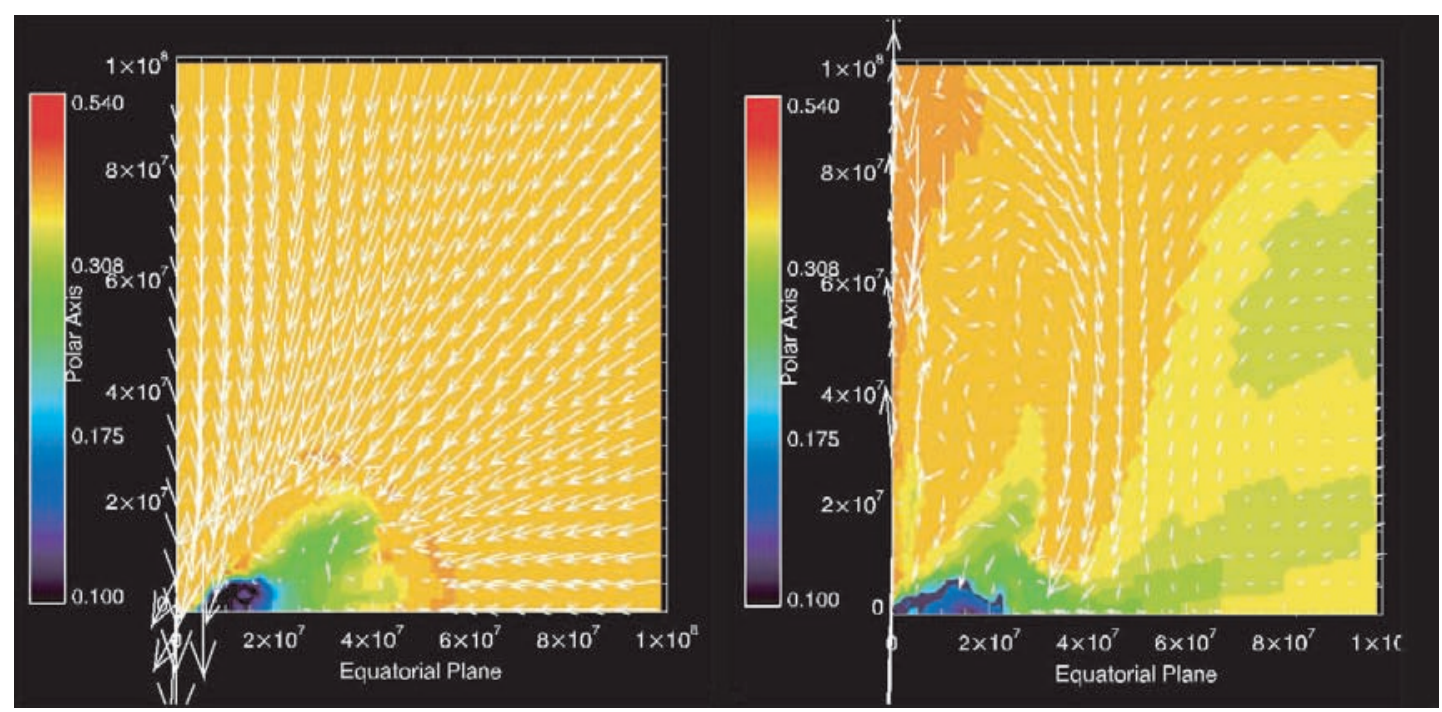

FIG. 18. - Contour of electron fraction with velocity fields for model 0 at $t=2.2 \mathrm{~s}($ left $)$ and model 9 at $t=2.2 \mathrm{~s}$ (right). The color represents the electron fraction on a linear scale $(0.1-0.540)$. 
innermost region of the accretion disk becomes optically thick against neutrinos and $\sim 10 \%-20 \%$ of neutrinos are absorbed.

The deposited energy by neutrino pair annihilation is of the order of $10^{49}-10^{50}$ ergs, which is much smaller than the explosion energy of a hypernova and a GRB. There are two ways to enhance the deposited energy by neutrino pair annihilation. One is to enhance the released gravitational energy; the other is to enhance the efficiency of energy deposition. The former corresponds to enhancing the mass accretion rate, which will be realized if effective angular momentum transfer is realized. From Figures 2, 8 , and 12 , it was inferred that magnetic fields seem to work efficiently so that a high mass accretion rate is realized. Of course, the mass accretion rate also depends on the distribution of initial angular momentum. We should investigate these effects further in the future. As for the efficiency of energy deposition, it will be enhanced when the general relativistic effects are taken into account. This is because neutrinos are trapped around the black hole, so that the possibilities of neutrino pair annihilation and neutrino capture become enhanced. This effect is investigated in detail by using a steady solution of an accretion disk in the forthcoming paper. Of course, we are planning to include this effect in our numerical code in the future.

Although we believe that our conclusion on the energetics mentioned above will be unchanged, we have to improve our treatment of the neutrino heating for further study. In this study we took the optically thin limit to estimate the neutrino heating rate. This will be justified by Figures 6 and 9 .

However, for further study, we have to investigate the cases in which mass accretion rate is higher than in this study to achieve an energetic explosion sufficient enough to explain the explosion energies of a hypernova and a GRB. In fact, we believe that the optically thin limit breaks down even for the models in this study at the highest density region. This is estimated as follows: The cross section of $\nu_{e}$ and $\bar{\nu}_{e}$ captures on free nucleons is given by $\sigma \sim \sigma_{0}\left(\epsilon_{\nu} / m_{e} c^{2}\right)^{2}$, where $\sigma_{0}=1.76 \times 10^{-44} \mathrm{~cm}^{2}$. Since the highest density in the accretion disk is of the order of $10^{12} \mathrm{~g} \mathrm{~cm}^{-3}$ at $r \sim 10^{6} \mathrm{~cm}$ (the scale height is also of the order of $10^{6} \mathrm{~cm}$ ) and typical energies of neutrinos are of the order of $10 \mathrm{MeV}$ (see Figs. 3 and 10), the optical depth at this region is $\tau=\sigma\left(\rho / m_{p}\right) L \sim$ $4.2\left(\epsilon_{\nu} / 10 \mathrm{MeV}\right)^{2}\left(\rho / 10^{12} \mathrm{~g} \mathrm{~cm}^{-3}\right)\left(L / 10^{6} \mathrm{~cm}\right)$. Thus, at the highest density region, the optically thin limit must break down. This picture is also confirmed in Figure $8 b$. In Figure $8 b$, as stated in $\S 3.2$, the energy deposition rate due to pair captures on free nucleons sometimes becomes larger than the neutrino luminosity. This reflects the fact that the optically thin limit breaks down at that time. Although we believe that our conclusion on the energetics will not be changed much, we are planning to develop the careful neutrino transfer code that includes emissions, absorptions, and scattering of neutrinos for further study. We also note four points that have to be improved for the treatments of neutrino heating. One is that we did not take into account the light crossing time of the system and assumed that the system is almost steady during the light crossing time when we estimate the neutrino heating rate. From Figures 5 and 7, the neutrino cooling and heating occur efficiently within several times $10^{7} \mathrm{~cm}$. Thus, the typical light crossing time will be of the order of $1 \mathrm{~ms}$. For comparison, the rotation period at the innermost region is $\sim 6.3 \times 10^{-4} \mathrm{~s}$. Since the system forms an accretion disk and the viscosity parameter $\alpha$ is 0.1 at most (Fig. 11), the system will be treated steady to at least 10 times the rotation period, $6.3 \times 10^{-3} \mathrm{~s}$. Thus, the treatment to neglect the light crossing time will be fairly justified. Second is that we update the neutrino heating rate every 100 time steps to save CPU time. The innermost radius is set to be $10^{6} \mathrm{~cm}$, so the typical time step is estimated to be $10^{6}(\Delta \theta / c) \mathrm{s}$, where $c$ is the speed of light and $\Delta \theta=\pi / 60$. Thus, 100 time steps correspond to $1.74 \sim$ $10^{-4} \mathrm{~s}$, which will be comparable to the free-fall timescale $\left[\tau_{\mathrm{ff}}=\right.$ $1 /(24 \pi G \rho)^{1 / 2} \sim 4.5 \times 10^{-4}\left(10^{12} \mathrm{~g} \mathrm{~cm}^{-3} / \rho\right)^{1 / 2} \mathrm{~s}$; Woosley 1986] and rotation period. Thus, we believe that this treatment will be fairly justified. Third, we have approximated that each neutrino energy spectrum due to each emission process is monotonic; that is, we assumed that only neutrinos with average energy are emitted when equation (15) is carried out. However, the cross section of neutrino pair annihilation is proportional to the square of the total energy in the center of mass, and that for electron-type neutrino absorption on free nucleons is proportional to the square of the neutrino's energy. Thus, the contribution of neutrinos with high energy will enhance the efficiency of neutrino heating.

These points will be improved when we can include a careful neutrino transfer code in the future. The last point is related with the nuclear reactions. In this study, the NSE was assumed for the region where $T \geq 5 \times 10^{9} \mathrm{~K}$. Thus, the reactions to maintain NSE occur suddenly when the temperature becomes so high as to satisfy the criterion. However, in reality, NSE might break down at the low-density region, where the cooling effect due to photodisintegration will be not as strong as in this simulation. It should be also noted that the photodissociation from He into nucleons is the strong cooling effect and absorbs thermal energy when this reaction is switched on. Thus, the thermal energy is suddenly absorbed by nuclear reactions. That is seen as the discontinuities of temperature in Figures 3 and 10.

Although we believe that these discontinuities do not change our conclusion on the energetics mentioned above (because many more neutrinos come from the inner region; Fig. 5), the profile of temperature will be solved smoothly when we use a nuclear reaction network instead of using the NSE relation. Since the emissivity of neutrinos depends very sensitively on the temperature (Bethe 1990; Herant et al. 1992; Lee \& Ramirez-Ruiz 2006), estimation of temperature should be treated carefully. We are planning to check the dependence of temperature on the nuclear reaction network and several EOSs in a forthcoming paper.

Finally, we discuss the detectability of neutrinos from collapsars. Since the event rate is much smaller than the normal core-collapse supernova, the chance probability to detect neutrino signals from a collapsar will be very small. However, if it occurs near our Galaxy, the neutrino signal from a collapsar will be distinguished from normal core-collapse supernovae. As for the normal corecollapse supernovae, the time evolution of the luminosity of neutrinos of each flavor is determined firmly by the binding energy of a neutron star and the opacity of a neutron star against neutrinos. On the other hand, in the case of a collapsar, the time evolution of neutrino luminosity will depend on the time evolution of the mass accretion rate, which in turn should depend on the initial distribution of angular momentum and magnetic fields. Thus, there should be many varieties of time evolution for the luminosity of neutrinos in the case of collapsars. Also, in the case of collapsars, the dominant process for generating neutrinos is pair captures on free nucleons (see Fig. 6), so in the case of a collapsar, the electron-type neutrinos will be produced much more frequently compared with other flavors. This is in contrast with the normal core-collapse supernovae (see, e.g., Buras et al. 2006 and references therein). Of course, we have to take vacuum and matter oscillation effects into account to estimate the spectrum of neutrinos from a collapsar precisely. In particular, in the case of a collapsar, the density distribution is far from spherically symmetric, so we have to be careful about the viewing angle for estimating the matter oscillation effect. It is true that the event rate of collapsars is smaller than normal core collapse supernovae, but the released gravitational energy can be larger if a considerable amount of 
mass of the progenitor falls into the central black hole (Nagataki et al. 2003a). Thus, we believe that there will be also a possibility to detect a neutrino background from collapsars.

\subsection{Effects of Magnetic Fields}

We have seen that the mass accretion rate seems to be enhanced in model 9, compared with model 0 (Fig. 2), which enhances the luminosity of neutrinos (Fig. 6) and energy deposition rate due to weak interactions (Fig. 8). This seems to be because magnetic viscosity is effective at the innermost region (Figs. 11 and 12) and multidimensional outflow (Fig. 1) carries angular momentum outward. Thus, amplification of magnetic fields is important not only for launching a jet by magnetic pressure but also for enhancing mass accretion rate and energy deposition rate through weak interactions. In our study we assume the axisymmetry of the system to save CPU time. In this case, the field built up by the effect of MRI decays due to Cowling's antidynamo theorem (Shercliff 1965). However, the plasma beta becomes lower than unity in the jet region (Fig. 14), which is embodied by the amplification of $B_{\phi}$ fields (Figs. 13 and 15). Thus, we believe that $B_{\phi}$ field is not amplified by MRI effects, but by winding up of poloidal fields due to differential rotation. The typical timescale of winding up at the innermost region will be (see Figs. 3 and 10)

$$
\tau_{\text {wind }} \sim 2 \pi \frac{d \ln r}{d \Omega} \sim \frac{2 \pi \ln 10}{10^{4}} \sim 1.45 \times 10^{-3} \mathrm{~s} .
$$

This timescale will correspond to the steep rising of energy of $B_{\phi}$ around $t=0.1 \mathrm{~s}$ in Figures 13 and 15 . After the steep growth, when the strength of $B_{\phi}$ becomes comparable to the poloidal component, the growth rate declines, since $B_{\phi}$ grows by winding the "weak" poloidal component (Takiwaki et al. 2004). The final time of the simulation is determined when the Alfvén speed reaches to the order of the speed of light. This is understood as follows: Alfvén crossing time in the $\theta$-direction at the innermost region becomes $r \Delta \theta / v_{\mathrm{A}}=10^{6}(\pi / 60) / c=1.74 \times 10^{-6} \mathrm{~s}$. Since the total time step is several times $10^{6}$, the final time is estimated to be several seconds, which is consistent with our results. The Alfvén speed is estimated to be

$$
\begin{aligned}
v_{\mathrm{A}}=\frac{B}{\sqrt{4 \pi \rho}} & \sim 2.82 \times 10^{8}\left(\frac{B}{10^{15} \mathrm{G}}\right)\left(\frac{10^{12} \mathrm{~g} \mathrm{~cm}^{-3}}{\rho}\right)^{1 / 2} \mathrm{~cm} \mathrm{~s}^{-1} \\
& \sim 2.82 \times 10^{10}\left(\frac{B}{10^{15} \mathrm{G}}\right)\left(\frac{10^{8} \mathrm{~g} \mathrm{~cm}^{-3}}{\rho}\right)^{1 / 2} \mathrm{~cm} \mathrm{~s}^{-1}
\end{aligned}
$$

which means that the final time is determined not by the time when the amplitude of $B_{\phi}$ reaches $10^{15} \mathrm{G}$ around the equatorial plane, but by the time when the amplitude of $B_{\phi}$ reaches $10^{15} \mathrm{G}$ at the low-density region, that is, around the polar region where the jet is launched.

As for $B_{r}$ and $B_{\theta}$ fields, from Figure 13, some instabilities seem to grow and saturate, which is similar to the behavior of MRI (Hawley \& Balbus 1991; Balbus \& Hawley 1998). At present, we consider these instabilities to be MRI modes with a wavelength of maximum growth mode unresolved. However, we cannot conclude that these instabilities are really unresolved MRI modes. This is due to the following reason: the dispersion relation of the linear MRI modes is obtained analytically by assuming that the accretion disk is supported by rotation (that is, in Kepler motion). On the other hand, as shown in Figures 3 and 10, the radial velocity is nonzero in the accretion disks in this study. Moreover, the radial flow speed is superslow magnetosonic (the speed of the slow magnetosonic wave is almost the same as that of the Alfvén wave in this study; Fig. 11). Since MRI is the instability of the slow magnetosonic waves in a magnetized and differentially rotating plasma, the dispersion relation may be changed considerably for such a superslow magnetosonic flow. However, there is no analytic solution for such a flow at present, so we use the dispersion relation of MRI for the discussion here.

Ignoring entropy gradients, the condition for the instability of the slow magnetosonic waves in a magnetized, differentially rotation plasma is (Balbus \& Hawley 1991)

$$
\frac{d \Omega^{2}}{d \ln r}+\left(\boldsymbol{k} \cdot \boldsymbol{v}_{\mathrm{A}}\right)^{2}<0,
$$

where $\boldsymbol{k}$ is the vector of the wavenumber. The wavelength of maximum growth of the linear instability is

$$
\begin{aligned}
\lambda_{0}= & \frac{2 \pi v_{\mathrm{A}}}{\Omega} \sim 1.77 \times 10^{3}\left(\frac{10^{4} \mathrm{~s}^{-1}}{\Omega}\right)\left(\frac{B}{10^{13} \mathrm{G}}\right) \\
& \times\left(\frac{10^{12} \mathrm{~g} \mathrm{~cm}^{-3}}{\rho}\right)^{1 / 2} \mathrm{~cm} .
\end{aligned}
$$

Since $\lambda_{0}$ is much smaller than the grid size of the innermost region $\left(\Delta r=3 \times 10^{5} \mathrm{~cm}\right)$, the linear MRI mode of maximum growth is not resolved in this study (note that the amplitudes of $B_{r}$ and $B_{\theta}$ are of the order of $10^{13} \mathrm{G}$ and much smaller than $B_{\phi}$; see Fig. 11). However, MRI grows as long as equation (19) holds. Since the value of the first term of equation (19) at the innermost region is roughly $-10^{8} / \ln 10 \sim-4.34 \times 10^{7}$, it is confirmed that the innermost region is unstable for the MRI mode with the wavelength longer than $\sim 10^{3}\left(B / 10^{13} \mathrm{G}\right)\left(10^{12} \mathrm{~g} \mathrm{~cm}^{-3} / \rho\right)^{1 / 2} \mathrm{~cm}$. The characteristic growing timescale is (Balbus \& Hawley 1998; Akiyama et al. 2003; Proga et al. 2003)

$$
\tau_{\text {MRI }} \sim 2 \pi\left|\frac{d \Omega^{2}}{d \ln r}\right|^{-1 / 2} \sim 6.74 \times 10^{-4} \mathrm{~s},
$$

which is seen in model 9 (Fig. 13) and model 8 (Fig. 15). The saturation level of $B_{r}$ seems to be slightly higher than that of $B_{\theta}$, which is similar to the results of the local simulations of MRI (Sano et al. 2004). Also, as shown in Figure 16, the growth rate of $B_{r}$ and $B_{\theta}$ components depends on the grid resolution, while the growth rate of $B_{\phi}$ does not depend on the grid resolution. In fact, the growth rate becomes smaller for a coarse mesh case (Fig. 16a). This is similar to the picture that $B_{r}$ and $B_{\theta}$ are amplified by MRI, while $B_{\phi}$ is amplified by the winding effect. The saturation level of $B_{r}$ and $B_{\theta}$ seems not to be sensitive to the grid resolution. As stated above, in order to prove firmly that $B_{r}$ and $B_{\theta}$ are amplified by MRI-like instability, the dispersion relation of linear growing modes for superslow magnetosonic flow has to be obtained analytically and has to be reproduced by numerical simulations with finer grid resolution, which is outside the scope of this study.

As shown in Figure 11, the estimated viscosity parameter becomes larger than $10^{-3}$ at almost all regions in $r \leq 4 \times 10^{4} \mathrm{~cm}$. Since the angular velocity becomes larger than $10^{3}$ for $r \leq 6 \times$ $10^{6} \mathrm{~cm}$ (Fig. 10), this viscosity becomes effective in a timescale of $1 \mathrm{~s}$, which is comparable to our simulations. Thus, we think that this viscosity drives the high mass accretion rate in the models with magnetic fields. However, as stated in $\S 3.2$, the angular momentum cannot be transferred to infinity along the radial 
direction. As shown in Figure 11, the flow is super-Alfvénic except for the innermost region. Thus, angular momentum cannot be conveyed outward by the Alfvén wave in the radial direction. At present, we believe that the outflow (including the jet) in the polar direction in model 9 should bring the angular momentum from the innermost region (see Fig. 1, bottom panels). This picture is similar to CDAF (Narayan et al. 2001). We are planning to present further analysis of this feature in a forthcoming paper.

Since the plasma beta can be of the order of unity at the innermost region (Fig. 14) and $B_{\phi}$ dominates $B_{r}$ and $B_{\theta}$ at the region $r \leq(7-8) \times 10^{7} \mathrm{~cm}$, we can understand that the magnetic pressure from $B_{r}$ and $B_{\theta}$ is much smaller than the radiation pressure (from photons, electrons, and positrons) and degenerate pressure. Thus, the jet cannot be launched by the effects of $B_{r}$ and $B_{\theta}$ only. The winding-up effect is necessary to amplify the $B_{\phi}$ field so that the magnetic pressure becomes comparable to the radiation and degenerate pressure. We saw that MRI-like instability seems to occur from the beginning of the simulations (see model 8 in Fig. 15). However, since the saturation level is not so high, the amplified energy of magnetic fields for $B_{r}$ and $B_{\theta}$ cannot be seen well at first (see model 9 in Fig. 13 and model 10 in Fig. 15). This is because at the early phase the steady accretion disk is not formed and there is not much released gravitational energy (see Fig. 2). In models 11 and 12, the initial total energies of magnetic fields are so high that the effect of MRI-like instability cannot be seen (Fig. 15).

As stated above, the axisymmetry of the system is assumed in this study. So the winding-up effect only amplifies the $m=0$ mode of $B_{\phi}$. Usually, it is pointed out that the saturation level of the winding-up effects becomes lower when three-dimensional simulations are performed (Hawley et al. 1995). Thus, $B_{\phi}$ may not be amplified as strong as $10^{15} \mathrm{G}$. Also, in the present study, the timescale for $B_{\phi}$ fields at the innermost region to be amplified to $\sim 10^{15} \mathrm{G}$ depends on the initial amplitude of the magnetic fields (see Figs. 13 and 15). However, if three-dimensional simulations are performed, instabilities due to MRI(-like) modes do not decay due to Cowling's antidynamo theorem. Also, the MRI(-like) modes with wavevectors whose $\phi$ components are nonzero, which amplify $B_{\phi}$, are included (Masada et al. 2006). If such MRI(-like) modes that amplify $B_{\phi}$ are included, the dependence of the dynamics on the initial amplitude of the magnetic fields may be diluted. We have to perform three-dimensional calculations to see what happens in more realistic situations.

As stated above, the time step becomes so small when the Alfvén crossing time becomes so small. Since this calculation is Newtonian, the speed of light is not included in the basic equations for macrophysics (eqs. [1]-[6]). In fact, we found that the Alfvén speed becomes larger than the speed of light at some points by a factor of 2 or so at the final stage of simulations. Thus, one of the solutions to overcome this problem is to develop the special relativistic MHD code, in which the Alfvén speed is, of course, solved to be smaller than the speed of light.

In this study we considered the ideal MHD without dissipation (of course, numerical viscosity is inevitably included due to finite gridding effects). When resistive heating is efficient, a considerable amount of energy in magnetic fields will be transferred to thermal energy by ohmic-like dissipation and reconnection, which will substantially change the dynamics of collapsars. The problem is, however, that the properties of resistivity of highdensity and high-temperature matter with strong magnetic fields are highly uncertain (it is noted that artificial resistivity is included in Proga et al. [2003] in order to account for the dissipation in a controlled way instead of allowing numerical effects to dissipate magnetic fields in an uncontrolled manner; see also Stone \& Pringle 2001).
Finally, we discuss the total explosion energy and bulk Lorentz factor of the jet. From Table 2, we can see that there seems to be a tendency that the mass of the jet becomes heavier when the initial amplitudes of the magnetic fields are stronger (models 10, 11 , and 12), although this tendency is not monotonic (models 8 and 9). This will reflect the fact that the jet is launched earlier and the density is still higher for a case with stronger initial magnetic fields. There also seems to be the tendency that the energy of the jet becomes larger when the initial amplitude of the magnetic fields is set to be stronger, although this tendency is not also monotonic (models 8 and 9). This tendency is not so remarkable, since the mass of the jet is greater for a stronger magnetic field case and the mass should have some amount of kinetic, thermal, and magnetic field energies. As for models 8 and 9, the information of the initial condition might be lost considerably since it takes much time to launch jets. From these results, we can conclude that no GRB jet is realized even if strong magnetic fields are assumed. We believe that there will be a possibility that a GRB jet is realized if we can perform numerical simulations for much longer physical time (say, $\sim 10-100 \mathrm{~s}$ ). In such long-timescale simulations, the energy of $B_{\phi}$ fields should be much more than $10^{50} \mathrm{ergs}$ (see Figs. 13 and 15). Also, the density in the jet may become lower with time because considerable mass will fall into the black hole along the polar axis. Thus, the terminal bulk Lorentz factor may be enhanced at a later phase. In order to achieve such a simulation, the special relativistic code will be helpful, as mentioned above.

\subsection{Prospect for Nucleosynthesis}

It is radioactive nuclei, ${ }^{56} \mathrm{Ni}$, and its daughter nuclei, ${ }^{56} \mathrm{Co}$, that brighten the supernova remnant and determine its bolometric luminosity. ${ }^{56} \mathrm{Ni}$ is considered to be synthesized through explosive nucleosynthesis because its half-life is very short (5.9 days). Thus, it is natural to conclude that explosive nucleosynthesis occurs in a hypernova that is accompanied by a GRB. However, it is not clearly known where the explosive nucleosynthesis occurs (e.g., Fryer et al. 2006a, 2006b).

Maeda et al. (2002) have performed a numerical calculation of explosive nucleosynthesis launching a jet by depositing thermal and kinetic energy at the innermost region. They have shown that a mass of ${ }^{56} \mathrm{Ni}$ sufficient to explain the observation of hypernovae $\left(\sim 0.5 M_{\odot}\right)$ can be synthesized around the jet region. In their calculation, all of the explosion energy was deposited initially. Thus, Nagataki et al. (2006) investigated the dependence of explosive nucleosynthesis on the energy deposition rate. They have shown that sufficient mass of ${ }^{56} \mathrm{Ni}$ can be synthesized as long as all of the explosion energy is deposited initially, while the synthesized mass of ${ }^{56} \mathrm{Ni}$ is insufficient if the explosion energy is deposited for $10 \mathrm{~s}$ (that is, the energy deposition rate is $10^{51} \mathrm{ergs} \mathrm{s}^{-1}$ ). This is because matter starts to move outward after the passage of the shock wave, and almost all of the matter moves away from the central engine before the injection of thermal energy $\left(=10^{52} \mathrm{ergs}\right)$ is completed, so the amount of mass remaining to completely burn, thereby synthesizing ${ }^{56} \mathrm{Ni}$, becomes little for such a long-duration explosion (see also Nagataki et al. 2003b).

On the other hand, the possibility is pointed out by some authors (MacFadyen \& Woosley 1999; Pruet et al. 2003) that a substantial amount of ${ }^{56} \mathrm{Ni}$ is produced in the accretion disk and part of it is conveyed outward by the viscosity-driven wind. However, there is much uncertainty as to how much ${ }^{56} \mathrm{Ni}$ is ejected from the accretion disk. This problem depends sensitively on the viscosity effects. Further investigation is required to estimate how much ${ }^{56} \mathrm{Ni}$ is ejected.

In this study we have found that the mass of ${ }^{56} \mathrm{Ni}$ in the accretion disk (see Fig. 4) at the final stage of simulations is of the 
order of $10^{-3} M_{\odot}\left(M_{\mathrm{Ni}}^{\text {tot }}\right.$ in Table 3$)$. Thus, if a considerable fraction of the synthesized ${ }^{56} \mathrm{Ni}$ is ejected without falling into the black hole, there will be a possibility supplying the sufficient amount of ${ }^{56} \mathrm{Ni}$ required to explain the luminosity of a hypernova. However, in the present study the ejected mass of ${ }^{56} \mathrm{Ni}$ was found to be only $10^{-11}$ to $10^{-6} M_{\odot}\left(M_{\mathrm{Ni}}^{\mathrm{esc}}\right.$ in Table 3$)$. This is because the entropy per baryon in the jet is so high (Fig. 17) that the light elements such as $n, p$, and He dominate in the jet. Thus, we could not show that sufficient mass of ${ }^{56} \mathrm{Ni}$ was ejected from the accretion disk in the present study. There will be two possibilities to extract enough ${ }^{56} \mathrm{Ni}$ from the accretion disk. One is that ${ }^{56} \mathrm{Ni}$ might be extracted efficiently from the accretion disk at a later phase. Since the typical temperature of the accretion disk will be lower when the mass of the central black hole becomes larger, the entropy per baryon in the jet will be decreased (Nagataki et al. 2003a). This feature should suggest that ${ }^{56} \mathrm{Ni}$ dominates in the jet component at the late phase. The other one is that some kinds of viscosities might work to convey efficiently the matter in the accretion disk outward. In MacFadyen \& Woosley (1999), they included $\alpha$-viscosity and showed that a considerable amount can be conveyed. Since $\alpha$-viscosity is not included in this study, such a feature was not seen. However, when three-dimensional simulations with higher resolution than in this study are done, many more modes of magnetic fields should be resolved, and some of them might be responsible for viscosities and work like $\alpha$-viscosity.

We discuss the possibility to synthesize heavy elements in collapsars. We have shown that neutron-rich matter with high entropy per baryon is ejected along the jet axis (Figs. 17 and 18). This is because electron capture dominates positron capture in the accretion disk (Fig. 6). Thus, there is a possibility that $r$-process nucleosynthesis occurs in the jet (Nagataki et al. 1997; Nagataki 2000, 2001; Nagataki \& Kohri 2001; Wanajo et al. 2002; Suzuki \& Nagataki 2005; Fujimoto et al. 2006; Nishimura et al. 2006). Moreover, we found that a mass element with high $Y_{e}(\sim 0.522)$ appears around the polar axis near the black hole. This is because $\nu_{e}$ capture dominates $\bar{\nu}_{e}$ capture at the region. This is because the flux of $\nu_{e}$ from the accretion disk (note that $\nu_{e}$ comes from electron capture) is sufficiently large to enhance $Y_{e}$ at the region. In such a high- $Y_{e}$ region, there will be a possibility that $r / p$-process nucleosynthesis occurs (Wanajo 2006). We are planning to perform such numerical simulations in the very near future. Also, there is a possibility that many neutrons are ejected from the jet, since the entropy per baryon amounts to the order of $10^{4}$. If so, there may be a possibility that signals from the neutron decays may be observed as the delayed bump of the light curve of the afterglow (Kulkarni 2005) or gamma rays (Razzaque \& Mészáros 2006b). Also, GeV emission may be observed by proton-neutron inelastic scattering (Mészáros \& Rees 2000; Rossi et al. 2006; Razzaque \& Mészáros 2006a). Finally, note that a careful treatment of neutrino transfer is important for the $r$ - and $r / p$-process nucleosynthesis. As stated in $\S 4.1$, the optically thin limit may break down at the high-density region. When the matter becomes opaque to neutrinos, the neutrinos become trapped and degenerate. In the high-density limit, the chemical equilibrium is achieved as $\mu_{e}+\mu_{p}=\mu_{n}+\mu_{\nu_{e}}$, where $\mu_{e}, \mu_{p}, \mu_{n}$, and $\mu_{\nu_{e}}$ are chemical potentials of electrons, protons, neutrons, and electron-type neutrinos, respectively. When the chemical potential of electron-type neutrinos is not negligible, the electron capture does not proceed further and the electron fraction does not decrease by much (Sato 1975), which should be crucial to the $r$ - and $r / p$-process nucleosynthesis.

\subsection{General Relativistic Effects}

In this section we discuss general relativistic effects that we are planning to include in our MHD simulation code.
First, we still believe that effects of energy deposition due to weak interactions (especially neutrino-antineutrino pair annihilation) can be a key process as the central engine of GRBs. In fact, the temperature of the accretion disk becomes higher especially for a Kerr black hole since the gravitational potential becomes deeper, much gravitational energy is released at the innermost region, and the radius of the innermost stable orbit becomes smaller for a Kerr black hole (Popham et al. 1999; MacFadyen \& Woosley 1999). This effect will enhance the luminosity of the neutrinos from the accretion disk (Popham et al. 1999) and the neutrino pair annihilation (Asano \& Fukuyama 2000; Miller et al. 2003; Kneller et al. 2006; Gu et al. 2006). In the vicinity of the black hole, most of the neutrinos and antineutrinos become shadows due to the bending effects of the neutrino geodesics. Since the shape and position of the black hole shadow depend on the physical parameters of the black hole (Bardeen 1973; Takahashi 2004, 2005), the effective area emitting most of the neutrino flux just outside of the black hole shadows is also determined by the black hole parameters. These effects are included only when geodesics and disk structures are precisely calculated.

According to our simulations, the plasma beta $\left(=p_{\text {gas }}+\right.$ radiation $/$ $\left.p_{\text {mag }}\right)$ in the polar region is lower than the plasma beta in other regions and the minimum value of the plasma beta is 0.193 . This means that the polar region is magnetically dominated. In such regions where the magnetic energy is dominated, such as a forcefree field, the extraction of the rotational energy of the black hole is expected as Poynting flux (Blandford \& Znajek 1977) and negative energy (Blandford \& Payne 1982; Takahashi et al. 1990). Although these effects have been confirmed numerically by many authors (Koide 2003; Mizuno et al. 2004b; Komissarov 2005; Hawley \& Krolik 2006; McKinney 2006), there are many unsolved issues on the central engine of GRBs. Especially, the neutrino radiation from the negative energy fluid in the realistic general relativistic accretion flow is one of the unsolved issues. The past studies roughly estimated that energy extraction due to the Blandford-Znajek process can be nonnegligible to explain the explosion energy of long GRBs (e.g., Lee et al. 2000a, 2000b; Di Matteo et al. 2002).

\subsection{Variety of Initial Conditions}

The properties of a progenitor of GRBs are still unknown. There are many uncertainties on mass-loss rate, final mass, angular momentum distribution, amplitude, and configuration of magnetic fields prior to collapse. These properties will depend not only on initial mass and metallicity of progenitors but also on the presence (or absence) and properties of companion stars (Maeder \& Meynet 2001; Woosley \& Heger 2006; MacFadyen et al. 2005). Thus, it is necessary to perform numerical simulations with many models of progenitors to ensure the validity of the mechanism to launch a GRB jet from progenitors. In particular, we believe that the initial angular momentum distribution should be important, since the mass accretion rate depends on it very sensitively.

\subsection{Prospects for Improvements of the Numerical Scheme}

As shown in equation (20), the critical wavelength of maximum growth of the linear MRI is quite short, so the adaptive mesh refinement method (e.g., Norman 2005; Zhang \& MacFadyen 2006; Morsony et al. 2006) is inevitably required to resolve the critical wavelength in a collapsar. Of course, much CPU time is required to perform three-dimensional calculations with the adaptive mesh refinement method. Moreover, when we try to include the neutrino transfer code, three dimensions are required additionally for the momentum space. However, people will be able 
to realize such an expensive simulation in the near future with the help of the rapidly growing power of supercomputers. Of course, we are planning to devote ourselves to developing such a numerical code.

\section{SUMMARY AND CONCLUSION}

We have performed two-dimensional MHD simulations by the ZEUS-2D code to investigate the dynamics of a collapsar that generates a GRB jet, taking account of realistic EOS (contribution of electrons, positrons, radiation, and ideal gas of nuclei), neutrino cooling and heating processes, magnetic fields, and gravitational force from the central black hole and self-gravity.

We have found that neutrino heating processes (neutrino and antineutrino pair annihilation, and $\nu_{e}$ and $\bar{\nu}_{e}$ captures on free nucleons) are not efficient enough to launch a jet. We have found that a jet is launched by magnetic fields (in particular, $B_{\phi}$ fields that are amplified by the winding-up effect). However, the ratio of total energy relative to the rest-mass energy in the jet at the final stage of simulations suggests that the bulk Lorentz factor of the jet will not reach as high as several hundred, so we conclude that the jet seen in this study will not be a GRB jet. We also found that the mass accretion rate seems to be enhanced in the models with magnetic fields. This might be because angular momentum is efficiently transferred by the viscosity due to the magnetic fields and multidimensional flow.

Since GRB jets are not obtained in this study, we believe that general relativistic effects, by which the efficiency of energy deposition through weak interactions will be enhanced and rotation energy of the black hole will be transferred to matter through the magnetic fields, will be important to generate a GRB jet. Thus, we are planning to develop a general relativistic MHD code in the very near future. Also, the accretion disk with magnetic fields may still play an important role in launching a GRB jet, and it may be seen whether we can perform numerical simulations for a much longer physical time (say, $\sim 10-100 \mathrm{~s}$ ). To realize such a simulation, the special relativistic code will be helpful because the Alfvén velocity is limited to the speed of light.

We have shown that a considerable amount of ${ }^{56} \mathrm{Ni}$ is synthesized in the accretion disk. Thus, if some fraction of the synthesized ${ }^{56} \mathrm{Ni}$ is ejected without falling into the black hole, there will be a possibility for the accretion disk to supply the sufficient amount of ${ }^{56} \mathrm{Ni}$ required to explain the luminosity of a hypernova. Also, we have shown that neutron-rich matter with high entropy per baryon is ejected along the rotation axis. This is because electron capture dominates positron capture. Moreover, we found that the electron fraction becomes larger than 0.5 around the polar axis near the black hole. This is because $\nu_{e}$ capture dominates $\bar{\nu}_{e}$ capture at the region. Thus, there will be a possibility that $r$-process and $r / p$-process nucleosynthesis occurs at these regions. Finally, many neutrons will be ejected from the jet, which suggests that signals from the neutron decays may be observed as the delayed bump of the light curve of the afterglow or gamma rays.

S. N. is grateful to S. Akiyama, R. Blandford, S. Fujimoto, S. Inutsuka, S. Mineshige, T. Sano, S. Yamada, T. Yamasaki, and M. Watanabe for useful discussions. The computation was carried out on NEC SX-5 and SX-8, SGI Altix3700 BX2, and Compaq Alpha Server ES40 at Yukawa Institute for Theoretical Physics, Kyoto University. This work is supported in part by a Grantin-Aid for the 21st Century COE "Center for Diversity and Universality in Physics" from the Ministry of Education, Culture, Sports, Science and Technology of Japan. S. N. is partially supported by Grants-in-Aid for Scientific Research from the Ministry of Education, Culture, Sports, Science and Technology of Japan through 16740134 . R. T. is partially supported by the Japan Society for the Promotion of Science 1710519. T. T is partially supported by the Japan Society for the Promotion of Science.
Akiyama, S., Wheeler, J. C., Meier, D. L., \& Lichtenstadt, I. 2003, ApJ, 584, 954

Aloy, M. A., Müller, E., Ibáñez, J. M., Martí, J. M., \& MacFadyen, A. 2000, ApJ, 531, L119

Asano, K., \& Fukuyama, T. 2000, ApJ, 531, 949

Balbus, S. A., \& Hawley, J. F. 1991, ApJ, 376, 214

. 1998, Rev. Mod. Phys., 70, 1

Bardeen, J. M. 1973, in Black Holes, ed. C. DeWitt \& B. DeWitt (New York: Gordon \& Breach), 215

Bethe, H. A. 1990, Rev. Mod. Phys., 62, 801

Blandford, R. D., \& Payne, D. G. 1982, MNRAS, 199, 883

Blandford, R. D., \& Znajek, R. L. 1977, MNRAS, 179, 433

Blinnikov, S. I., Dunina-Barkovskaya, N. V., \& Nadyozhin, D. K. 1996, ApJS, 106,171

Bloom, J. S., Kulkarni, S. R., \& Djorgovski, S. G. 2002, AJ, 123, 1111

Bloom, J. S., et al. 1999, Nature, 401, 453

Buras, R., Rampp, M., Janka, H.-Th., \& Kifonidis, K. 2006, A\&A, 447, 1049

Cooperstein, J., van den Horn, L. J., \& Baron, E. A. 1986, ApJ, 309, 653

Di Matteo, T., Perna, R., \& Narayan, R. 2002, ApJ, 579, 706

Epstein, R. I., \& Pethick, C. J. 1981, ApJ, 243, 1003

Fryer, C. L., Herwig, F., Hungerford, A., \& Timmes, F. X. 2006a, ApJ, 646, L131

Fryer, C. L., \& Mészáros, P. 2003, ApJ, 588, L25

Fryer, C. L., Young, P. A., \& Hungerford, A. L. 2006b, ApJ, 650, 1028

Fujimoto, S., Kotake, K., Yamada, S., Hashimoto, M., \& Sato, K. 2006, ApJ, 644,1040

Galama, T. J., et al. 1998, Nature, 395, 670 2000, ApJ, 536, 185

Garnavich, P. M., et al. 2003, ApJ, 582, 924

Goodman, J., Dar, A., \& Nussinov, S. 1987, ApJ, 314, L7

Gorosabel, J., et al. 2003, A\&A, 409, 123

Gu, W.-M., Liu, T., \& Lu, J.-F. 2006, ApJ, 643, L87

Hawley, J. F., \& Balbus, S. A. 1991, ApJ, 376, 223

Hawley, J. F., Gammie, C. F., \& Balbus, S. A. 1995, ApJ, 440, 742
Hawley, J. F., \& Krolik, J. H. 2006, ApJ, 641, 103

Heger, A., Langer, N., \& Woosley, S. E. 2000, ApJ, 528, 368

Herant, M. E., Benz, W., \& Colgate, S. A. 1992, ApJ, 395, 642

Herant, M. E., Benz, W., Hix, W. R., Fryer, C. L., \& Colgate, S. A. 1994, ApJ, 435,339

Hjorth, J., et al. 2003, Nature, 423, 847

Igumenshchev, I. V., \& Abramowicz, M. A. 2000, ApJS, 130, 463

Itoh, N., Adachi, T., Nakagawa, M., Kohyama, Y., \& Munakata, H. 1989, ApJ, 339,354

Iwamoto, K., et al. 1998, Nature, 395, 672

Kneller, J. P., McLaughlin, G. C., \& Surman, R. A. 2006, J. Phys. G, 32, 443

Koide, S. 2003, Phys. Rev. D., 67, 104010

Komissarov, S. S. 2005, MNRAS, 359, 801

Kotake, K., Yamada, S., \& Sato, K. 2003, ApJ, 595, 304

Kulkarni, S. R. 2005, preprint (astro-ph/0510256)

Lee, H. K., Brown, G. E., \& Wijers, R. A. M. J. 2000a, ApJ, 536, 416

Lee, H. K., Wijers, R. A. M. J., \& Brown, G. E. 2000b, Phys. Rep., 325, 83

Lee, W. H., \& Ramirez-Ruiz, E. 2006, ApJ, 641, 961

MacFadyen, A. I., Ramirez-Ruiz, E., \& Zhang, W. 2005, BAAS, 37, 1410

MacFadyen, A. I., \& Woosley, S. E. 1999, ApJ, 524, 262

Maeda, K., Nakamura, T., Nomoto, K., Mazzali, P. A., Patat, F., \& Hachisu, I. 2002, ApJ, 565, 405

Maeder, A., \& Meynet, G. 2001, A\&A, 373, 555

Masada, Y., Sano, T., \& Takabe, H. 2006, ApJ, 641, 447

Mazzali, P. A., et al. 2006, Nature, 442, 1018

McKinney, J. C. 2006, MNRAS, 368, 1561

Mészáros, P., \& Rees, M. 2000, ApJ, 541, L5

Miller, W. A., George, N. D., Kheyeets, A., \& McGhee, J. M. 2003, ApJ, 583, 833

Mirabal, N., Halpern, J. P., An, D., Thorstensen, J. R., \& Terndrup, D. M. 2006, ApJ, 643, L99

Mizuno, Y., Yamada, S., Koide, S., \& Shibata, K. 2004a, ApJ, 606, 395

2004b, ApJ, 615, 389 
Morsony, B. J., Lazzati, D., \& Begelman, M. C. 2006, ApJ, submitted (astro$\mathrm{ph} / 0609254)$

Nagataki, S. 2000, ApJS, 127, 141 2001, ApJ, 551, 429

Nagataki, S., Hashimoto, M., Sato, K., \& Yamada, S. 1997, ApJ, 486, 1026

Nagataki, S., \& Kohri, K. 2001, PASJ, 53, 547

Nagataki, S., Kohri, K., Ando, S., \& Sato, K. 2003a, Astropart. Phys., 18, 551

Nagataki, S., Mizuta, A., \& Sato, K. 2006, ApJ, 647, 1255

Nagataki, S., Mizuta, A., Yamada, S., Takabe, H., \& Sato, K. 2003b, ApJ, 596, 401

Narayan, R., Piran, T., \& Kumar, P. 2001, ApJ, 557, 949

Nishimura, S., Kotake, K., Hashimoto, M., Yamada, S., Nishimura, N., Fujimoto, S., \& Sato, K. 2006, ApJ, 642, 410

Norman, M. L. 2005, in Adaptive Mesh Refinement: Theory and Applications, ed. T. Plewa, T. Linde, \& V. G. Weirs (Berlin: Springer)

Paczyński, B., \& Witta, P. J. 1980, A\&A, 88, 23

Popham, R., Woosley, S. E., \& Fryer, C. 1999, ApJ, 518, 356

Price, P. A., et al. 2003, Nature, 423, 844

Proga, D. 2005, ApJ, 629, 397

Proga, D., \& Begelman, M. C. 2003, ApJ, 582, 69

Proga, D., MacFadyen, A. I., Armitage, P. J., \& Begelman, M. C. 2003, ApJ, 599, L5

Pruet, J., Woosley, S. E., \& Hoffman, R. D. 2003, ApJ, 586, 1254

Razzaque, S., Mészáros, P. 2006a, ApJ, 650, 998

2006b, J. Cosmol. Astropart. Phys., 0606, 006

Reichart, D. E. 1999, ApJ, 521, L111

Rockefeller, G., Fryer, C. L., \& Li, H. 2006, ApJ, submitted (astro-ph/0608028)

Rossi, E. M., Beloborodov, A. M., \& Rees, M. J. 2006, MNRAS, 369, 1797

Rosswog, S., \& Liebendörfer, M. 2003, MNRAS, 342, 673
Rybicki, G. B., \& Lightman, A. P. 1979, Radiative Process in Astrophysics (New York: Wiley)

Sano, T., Inutsuka, S., Turner, N. J., \& Stone, J. M. 2004, ApJ, 605, 321

Sato, K. 1975, Prog. Theor. Phys., 54, 1325

Sekiguchi, Y., \& Shibata, M. 2005, Phys. Rev. D, 71, 084013

Shercliff, J. A. 1965, A Textbook of Magnetohydrodynamics (Oxford: Pergamon)

Shibata, M., \& Sekiguchi, Y. 2005, Phys. Rev. D, 71, 024014

Stanek, E. Z., et al. 2003, ApJ, 591, L17

Stone, J. M., \& Norman, M. L. 1992a, ApJS, 80, 753 1992b, ApJS, 80, 791

Stone, J. M., \& Pringle, J. E. 2001, MNRAS, 322, 461

Suzuki, T. K., \& Nagataki, S. 2005, ApJ, 628, 914

Takahashi, K., El Eid, M. F., \& Hillebrandt, W. 1978, A\&A, 67, 185

Takahashi, M., Nitta, S., Tatematsu, Y., \& Tomimatsu, A. 1990, ApJ, 363, 206

Takahashi, R. 2004, ApJ, 611, 996 2005, PASJ, 57, 273

Takiwaki, T., Kotake, K., Nagataki, S., \& Sato, K. 2004, ApJ, 616, 1086

Thielemann, F.-K., Nomoto, K., \& Hashimoto, M. 1996, ApJ, 460, 408

Vreeswijk, P. M., Fender, R. P., Garrett, M. A., Tingay, S. J., Fruchter, A. S., \&

Kaper, L. 2001, A\&A, 380, L21

Wanajo, S. 2006, ApJ, 647, 1323

Wanajo, S., Itoh, N., Ishimaru, Y., Nozawa, S., \& Beers, T. C. 2002, ApJ, 577, 853

Woosley, S. E. 1986, in Nucleosynthesis and Chemical Evolutions, ed. B.

Hauck, A. Maeder, \& G. Meynet (Geneva: Geneva Obs.), 1 1993, ApJ, 405, 273

Woosley, S. E., Eastman, R. G., \& Schmidt, B. P. 1999, ApJ, 516, 788

Woosley, S. E., \& Heger, A. 2006, ApJ, 637, 914

Zhang, W., \& MacFadyen, A. I. 2006, ApJS, 164, 255 